\title{
MOF-Based Nanoagent Enables NIR-Triggered Dual Damage to Mitochondria via Synergistically Reinforced Oxidative Stress and Calcium Overload
}

Zhiyuan Tian ( $\nabla$ zytian@ucas.ac.cn )

University of Chinese Academy of Sciences (UCAS) https://orcid.org/0000-0002-1436-6818

\section{Weier Bao}

University of Chinese Academy of Sciences

Ming Liu

University of Chinese Academy of Sciences

Jiaqi Meng

University of Chinese Academy of Sciences

Siyuan Liu

University of Chinese Academy of Sciences

Shuang Wang

Institute of Process Engineering, Chinese Academy of Sciences

\section{Rongrong Jia}

Shanghai Tongren Hospital, Shanghai Jiao Tong University School of Medicine

\section{Yugang Wang}

Shanghai Tongren Hospital, Shanghai Jiao Tong University School of Medicine

\section{Guanghui Ma}

State Key Laboratory of Biochemical Engineering, Institute of Process Engineering, Chinese Academy of Sciences https://orcid.org/0000-0001-9154-5556

\section{Wei Wei}

Institute of Process Engineering https://orcid.org/0000-0002-6244-3187

\section{Article}

Keywords: MOFs, mitochondrial, photoacidification, UCNP, anticancer

Posted Date: April 15th, 2021

DOI: https://doi.org/10.21203/rs.3.rs-379232/v1

License: (c) (1) This work is licensed under a Creative Commons Attribution 4.0 International License.

Read Full License 
Version of Record: A version of this preprint was published at Nature Communications on November 4th, 2021. See the published version at https://doi.org/10.1038/s41467-021-26655-4. 


\section{Abstract}

We herein developed a core-shell type antitumor nanoagent based on the synergistically reinforced oxidative stress and calcium overload to mitochondria, both of which were triggered by near-infrared (NIR) light. The folic acid (FA) moiety decorated on MOF shells enabled efficient cellular uptake of nanoagents. The upconversion nanoparticle (UCNP) core converted NIR light to ultraviolet (UV) light with the latter catalyzed $\mathrm{Fe}^{3+}$-to- $\mathrm{Fe}^{2+}$ reduction and simultaneously activated the photoacid generator encapsulated in the cavities of MOFs, which enabled the release of free $\mathrm{Fe}^{2+}$ and photoacidification of intracellular microenvironment, respectively. The overexpressed $\mathrm{H}_{2} \mathrm{O}_{2}$ in the mitochondria, highly reactive $\mathrm{Fe}^{2+}$ and acidic milieu synergistically reinforced Fenton reactions for producing lethal hydroxyl radicals in mitochondria. Moreover, the photoacidification of plasma induced calcium influx, leading to calcium overload in the mitochondria. The therapeutic potency of the nanoagent based on the dual mitochondrial damage has been unequivocally confirmed in cell- and patient-derived tumor xenograft models in vivo.

\section{Introduction}

Despite remarkable advancements, mainstay anticancer strategies still encounter multifaceted challenges in terms of their limited effectiveness in the clinic. For instance, chemotherapy, the standard treatment strategy for patients with advanced stages of tumor progression, mostly involves modulation of the cell cycle and typically suffers from serious side effects and drug resistance ${ }^{1-4}$. To date, many evidences have revealed that mitochondria play key multifunctional roles in oncogenesis, including adenosine triphosphate (ATP) generation, redox and calcium homeostasis, and metabolic signal transduction $^{5-7}$. Moreover, it has been well established that mitochondria in tumor cells are characterized by distinctive properties, including hypoxia and upregulated hydrogen peroxide $\left(\mathrm{H}_{2} \mathrm{O}_{2}\right)^{8,9}$. Thus, an opportunity has risen to develop new antitumor agents by targeting mitochondria and utilizing their inherent properties as crucial triggering factors, which is expected to confine the range of action locally to the tumors and therefore present anticancer capabilities with the desired specificity and efficacy.

Among emerging antitumor therapeutics, chemodynamic therapy (CDT) based on oxidative stress holds unequivocal superiority in terms of matching with the abovementioned design rationale for mitochondrial damage ${ }^{10}$. Specifically, CDT on the basis of the Fenton reaction or Fenton-like reactions can be facilitated in the mitochondrial microenvironment due to the upregulation of the $\mathrm{H}_{2} \mathrm{O}_{2}$ substrate ${ }^{8,9}$. Moreover, as the crucial species involved in $\mathrm{CDT}$, the $\cdot \mathrm{OH}$ is characterized by much stronger oxidative stress than its counterpart active species involved in photodynamic therapy (PDT), namely, ${ }^{1} \mathrm{O}_{2}{ }^{11,12}$. Additionally, $\mathrm{CDT}$ circumvents the intrinsic impediments originating from the hypoxia that PDT typically encounters ${ }^{13}$.

Although CDT holds great promise for antitumor treatment based on mitochondrial damage, some hurdles regarding its practicality still exist. Firstly, the Fenton reaction prefers an acidic environment, with an optimal reaction $\mathrm{pH}$ ranging from 2.0 to $5.0^{14}$, and the intracellular $\mathrm{pH}$ of tumor predominantly exhibited $\sim 7.4^{15}$, which is not acidic enough to enable an efficient Fenton reaction and the accumulation 
of sufficient $\cdot \mathrm{OH}$. Furthermore, because $\mathrm{Fe}^{2+}$ is typically more productive in generating $\cdot \mathrm{OH}$ via the Fenton reaction but more unstable than $\mathrm{Fe}^{3+16,17}$, efficient delivery of the Fe component into the tumor cytoplasm and the on-demand release of sufficient $\mathrm{Fe}^{2+}$ in situ remain a challenge. Additionally, for improved therapeutic effectiveness via additive or even synergistic effects, the challenge of simultaneously administration of combined approaches based on different pathways involved in mitochondrial damage also remains.

We herein developed a new type of metal-organic framework (MOF)-based core-shell nanoagent with NIRtriggered potent antitumor capability based on the combination of Fenton reaction-enabled oxidative stress and an imbalance in calcium homeostasis, both of which contribute to mitochondrial damage. Such a type of nanoagent (denoted as FMUP) was fabricated via the one-pot self-assembly of a MOF (MIL-100, denoted as M) shell around an up-conversion nanoparticle (UCNP) core (denoted as U). The MOFs shell was constructed using $\mathrm{Fe}^{3+}$ as central metal ion while 1, 3, 5-benzenetricarboxylic acid (BTC) and a small portion of folic acid (FA, denoted as F) as bridging ligands. Additionally, the photoacid generator (4-(2-(4-hydroxy-3,5- dimethoxyphenyl)-2-oxoethoxy)-4-oxobutan-1-aminium) (pHP, denoted as P) was encapsulated into the cavities of the MOF units. FA decorated onto the MOFs is expected to facilitate accumulation of the nanoagents at tumor sites via the overexpression of folate receptors (FRs) on tumor cells. The UCNP core is capable of converting NIR photons into UV photons ${ }^{18,19}$ and is expected to play crucial roles in two key processes: i) the generation of UV light to mediate the reduction of $\mathrm{Fe}^{3+}$ to $\mathrm{Fe}^{2+20}$, and $\mathrm{ii}$ ) the transfer of excitation energy to photoacid generators within the close proximity to consequently trigger the release of $\mathrm{H}^{+}$from the latter (Scheme 1).

Following the first process, the MOF units are expected to disintegrate owing to the change in chelation and release free $\mathrm{Fe}^{2+}$ from the node sites of the framework ${ }^{21,22}$. As a result of the second process, the desired increase in the intralysosomal concentration of $\mathrm{H}^{+}$is expected. With sharp increases in intralysosomal concentration $\left(\mathrm{Fe}^{2+}\right.$ and $\mathrm{H}^{+}$) and therefore osmotic pressure, the lysosomes are expected to burst and release $\mathrm{Fe}^{2+}$ and $\mathrm{H}^{+}$into the cytoplasm. These NIR-triggered processes are expected to synergistically reinforce the efficiency of the Fenton reaction by presenting more productive reactants and the optimal reaction condition (an acidic environment) to therefore generate augmented oxidative stress in the $\mathrm{H}_{2} \mathrm{O}_{2}$-abundant mitochondria. As an accompanying effect associated with photoacidification, an increase in the acidity of the intratumoral cytoplasm will markedly give rise to calcium influx causing mitochondrial calcium overload ${ }^{23-27}$. The outstanding therapeutic efficacy of FMUP based on the abovementioned NIR-triggered dual damage to mitochondria was unequivocally verified in various tumor cell lines in vitro, cell derived tumor xenograft (CDX) and patient derived tumor xenograft (PDX) models in vivo, proclaiming its potential as a safe and potent anticancer agent based on efficiently amplified mitochondrial damage.

\section{Scheme 1. Schematic Illustration of the construction of FMUP nanoagent and the underlying anticancer}

mechanism. (A) FMUP nanoagent containing UCNP as the core and photoacid (pHP) encapsulated in the FA-doped nanoagent shell by one-pot self-assembly. The FMUP nanoagent was synthesized by 
coordination of carboxyl groups on BTC and FA with $\mathrm{Fe}^{3+}$. The UCNP as the core was located in the shell and simultaneously the pHP was loaded in the pore of the nanoagent. (B) Corresponding anticancer mechanism of FMUP nanoagent. $\triangle$ Active internalization of FMUP in tumor cells helped by FA functionalization. $\mathbb{Q}$ Lysosome escape of FMUP induced by the increase of osmotic pressure after NIR light irradiation. $\otimes \mathrm{Fe}^{2+}$ and $\mathrm{H}^{+}$release from FMUP upon NIR light irradiation. $\otimes$ Photoacidification of intracellular microenvironment induced calcium influx and therefore calcium overload in the mitochondria and simultaneously generated a key acidic environment for efficient Fenton reactions. The release of Fe $\mathrm{F}^{2+}$ and photoacidification synergistically reinforced Fenton reactions and therefore produced a large number of $\cdot \mathrm{OH}$ within the close proximity of mitochondria. $\otimes$ As a result, the calcium overloaded and plentiful $\cdot \mathrm{OH}$ enabled dual damage to mitochondria and further induced cell death. (see Supplemental Materials for Scheme 1)

\section{Results}

\section{Construction and characterization of FMUP nanoagents.}

Our FMUP nanoagents were fabricated via the self-assembly of $\mathrm{Fe}^{3+}$ and polydentate bridging ligands (BTC and FA) around the UCNP core according to the literature procedure with minor modifications (Fig. $1 \mathrm{~A})$. During this process, the pHP generator molecules were incorporated within the cavities of the MOF (Fig. S1). The as-prepared nanoagents exhibited an average diameter of $\sim 130 \mathrm{~nm}$ and a typical core-shell structure with the UCNP component located at the core cloaked by the MOF shell (Fig. 1B). This unique structure was further confirmed by the line scanning (Fig. 1C) and energy dispersive X-ray data obtained from the elemental mapping of FMUP (Fig. S2). Owing to its partial participation in chelation with $\mathrm{Fe}^{3+}$ ions (Fig. S3), the FA moiety was successfully decorated onto the MOF units, which could be verified by the appreciable influence on the size and surface properties (Fig. 1D). Specifically, FMUP displayed a slightly increased size and decreased zeta potential compared to the pristine MUP, which can be attributed to the decoration of the FA moiety onto the surface of the MOF nanostructure. In terms of pHP, the loading amount was calculated to be up to $12.61 \%$ wt (Fig. S4).

With FMUP in hand, we further tested its stability. During one week of storage in cell culture medium without fetal bovine serum (FBS), FMUP did not display obvious changes in its hydrodynamic radius or zeta potential, suggesting favorable stability for intravenous injection (Fig. 1E). We also estimated the potential of such Fe-containing nanoagents as $\mathrm{T}_{2}$-weighted magnetic resonance imaging (MRI) contrast agents. As illustrated in Fig. $1 \mathrm{~F}$, the relaxation rate $\left(1 / \mathrm{T}_{2}\right)$ of water protons markedly increased from approximately 2.7 to $49.7 \mathrm{~s}^{-1}$, and the grey level of the $T_{2}$-weighted images was significantly augmented upon increasing the concentration of Fe from 0.008 to $0.348 \mathrm{mM}$, indicating the sufficiently strong magnetism of FMUP for in vivo MRI use and its potential for image-guided anticancer therapy.

\section{Response capability of FMUP to NIR light}


As mentioned above, the design rationale for the nanoagent is based on the UCNP-mediated NIR-to-UV excitation energy conversion and subsequent UV light-mediated reduction of $\mathrm{Fe}^{3+}$ to $\mathrm{Fe}^{2+}$ and release of $\mathrm{H}^{+}$from the photoacid generator component (Fig. 1G). Upon irradiation with $980-\mathrm{nm}$ laser $\left(1.0 \mathrm{~W} / \mathrm{cm}^{2}, 5\right.$ min), we clearly observed that the FMUP-dispersed aqueous sample displayed strong emission with a peak at approximately $365 \mathrm{~nm}$ due to the encapsulated UCNP (Fig. S5). Moreover, the MOF-based coreshell nanostructures obviously disintegrated and aggregated in FMUP-L group (Fig. 1H), resulting in apparently marked increase in the size of the nanostructure with a much wider distribution (Fig. 1I). The above disintegration of FMUP could be assigned to the abrupt change in chelation of the central metal ions with ligands upon the NIR-to-UV triggered reduction. As shown in Fig. $1 \mathrm{~J}$, the evolution of both $\mathrm{Fe}^{3+}$ and $\mathrm{Fe}^{2+}$ content within the composite nanostructures as a function of NIR light irradiation time clearly indicates the increase in $\mathrm{Fe}^{2+}$ ions at the expense of $\mathrm{Fe}^{3+}$. Notably, the contents of $\mathrm{Fe}^{3+}$ and $\mathrm{Fe}^{2+}$ mirror each other, with a decrease in $\mathrm{Fe}^{3+}$ that is proportional to the increase in $\mathrm{Fe}^{2+}$, suggesting a clean photocatalyzed one-to-one conversion with high efficiency.

In addition to $\mathrm{Fe}^{2+}$ release, another outcome of the NIR-to-UV conversion was the photocatalysis of pHP to acidify the microenvironment ${ }^{28,29}$. For verification, we evaluated the acidity evolution of the FMUP suspension upon irradiation with $980-\mathrm{nm}$ laser $\left(1.0 \mathrm{~W} / \mathrm{cm}^{2}\right)$ using a SNARF ${ }^{\circledR}-1$ probe. As shown in Fig. $1 \mathrm{~K}$, the $\mathrm{pH}$ of the sample rapidly decreased from weak alkalinity to weak acidity within $10 \mathrm{~min}$. Together with the released $\mathrm{Fe}^{2+}$, such acidification could significantly accelerate the Fenton reaction. To test this aspect, we used 5, 5-dimethyl-1-pyrroline-oxide (DMPO) as a $\cdot \mathrm{OH}$ trapping agent for ESR spectral characterization (Fig. 1L). The yield of $\cdot \mathrm{OH}$ in the FMUP-L group was much higher than that in the FMU-L and FMUP groups, again indicating the synergistic effect of $\mathrm{Fe}^{2+}$ and acidification on reinforcing $\cdot \mathrm{OH}$ production. Similar results were also obtained via a bleaching experiment with methylene blue (MB) (Fig. S6). These results together indicated the ideal response ability of FMUP to NIR light as well as the desired outcomes of $\cdot \mathrm{OH}$ production.

\section{Intracellular fate of FMUP}

Owing to the overexpressed FA receptors on the tumor membrane ${ }^{30,31}$, functionalization of carriers with FA moieties has been proven to be very effective for circumventing the problem of low targeting efficiency to the tumor site. To evaluate the FA-enabled targeting performance, HeLa cells were incubated with Cy5labelled FMUP (with FA) or MUP (without FA), and confocal laser scanning microscopy (CLSM) images were captured (Fig. 2A). As expected, HeLa cells treated with FMUP presented much stronger red fluorescence than cells treated with MUP. These cells were also detected by flow cytometry for quantification (Fig. S7), and the internalization amount of FMUP was more than 2.9 folds that of MUP, again verifying that FA decoration significantly improved internalization by HeLa cells.

After internalization, most foreign nanocarriers are restricted to mature lysosomes. However, ideal mitochondria damage-targeting potency is expected if the Fenton reaction occurs in close proximity to the mitochondria because of the upregulation of $\mathrm{H}_{2} \mathrm{O}_{2}$ in the mitochondria and the extremely short 
diffusion distance of the $\cdot \mathrm{OH}$ species $^{32,33}$. To test whether our FMUP could break through the lysosomal compartmentalization, we evaluated the co-localization of FMUP (labelled with Cy5) and lysosomes (labelled with LysoTracker ${ }^{\text {TM }}$ Green) inside HeLa cells (Fig. 2B). Specifically, the co-localization rate of FMUP with lysosomes dramatically decreased from $70.34 \%$ to $10.38 \%$ upon irradiation with 980 -nm laser $\left(1.0 \mathrm{~W} / \mathrm{cm}^{2}, 5 \mathrm{~min}\right)$. Moreover, the TEM images provided direct evidence that FMUP resided in lysosomes, while lysosome deconstruction was observed in the FMUP-L group. The enabling factor for such efficient lysosomal escape was plausibly due to the high osmotic pressure originating from the sharply upregulation of $\mathrm{Fe}^{2+}$ and $\mathrm{H}^{+}$in the lysosomes that FMUP caused upon NIR light stimulus.

\section{Intracellular acidification and the dual mitochondrial effects}

Next, the capability of FMUP nanoagents to cause intracellular acidification was evaluated using $\mathrm{SNARF}^{\circledR}-1$ as a pH probe (Fig. 2C). Owing to the efficiently generated $\mathrm{H}^{+}$from pHP upon irradiation of 980-nm laser, HeLa with internalized FMUP emitted vivid green fluorescence after irradiation, indicating an acidified cytoplasm. By using FMU-L as a control, we observed that the fluorescence of HeLa cells did not change to green due to the absence of the photoacid generator. For detailed quantification, the intracellular $\mathrm{pH}$ after different treatments was determined from the calibration curves constructed earlier from the CLSM analysis. Notably, the intracellular pH value in the FMUP-L group appreciably decreased to $\sim 5.5$ but was still approximately neutral in the FMU-L group, further verifying the crucial role of the photoacid generator for the desired intracellular acidification.

Given that abnormal intracellular acidity generally leads to an imbalance in intracellular calcium ion ${ }^{24,27}$, we continued to investigate the evolution of intracellular $\mathrm{Ca}^{2+}$ levels in HeLa cells using Fluo-3 as a fluorescent calcium probe (Fig. 2D). When the cells were incubated with FMUP or FMU, no noticeable increase in intracellular $\mathrm{Ca}^{2+}$ levels prior to NIR light irradiation was observed. In sharp contrast, 5-min irradiation of 980-nm laser unequivocally generated a difference in cytoplasmic $\mathrm{Ca}^{2+}$ levels with the probe in FMUP-internalized cells displaying clearly stronger fluorescence than that in FMU-internalized cells and the discrepancy in fluorescence brightness in two cases appreciably increasing up to a maximum level $\sim 25 \mathrm{~min}$ after the irradiation. As a result of such photoacidification-enabled upregulation of intracellular $\mathrm{Ca}^{2+}$ level, increase in the mitochondrial $\mathrm{Ca}^{2+}$ level was also observed. Specifically, it was found that the FMUP-L group clearly displayed much higher expression level of mitochondrial calcium uniporter (MCU) ${ }^{34-36}$ than that the FMU-L group (Fig. 2E).

In addition to calcium overload in the mitochondria, the observed NIR-triggered release of $\mathrm{Fe}^{2+}$ and $\mathrm{H}^{+}$ also prompted us to investigate the influence on intracellular $\cdot \mathrm{OH}$ generation by using 2 ', 7 'dichlorofluorescin diacetate (DCFH-DA) as a probe (Fig. 2F). Considering that multiple factors were probably involved in the Fenton reaction, we investigated HeLa cells upon treatments with a series of formulations (PBS, MU, MU-L, FMU-L, FMUP-L, FMUP-L+Lip-1) for comparison. Compared to the MU group with almost no fluorescence, the cells in the MU-L group exhibited weak fluorescence, which verified the NIR light-assisted reduction of $\mathrm{Fe}^{3+}$ to $\mathrm{Fe}^{2+}$ for the improved Fenton reaction. Owing to the 
increase in internalization mediated by the FA ligand, a further increased fluorescence, which indicated intracellular $\cdot \mathrm{OH}$ concentration, was observed in the FMU-L group. Upon further photoacidification that pHP enabled, cells in the FMUP-L group indeed exhibited the brightest fluorescence, indicating the generation of considerable amount of $\cdot \mathrm{OH}$ species from Fenton reactions. Considering the abundant $\mathrm{H}_{2} \mathrm{O}_{2}$ in the mitochondria ${ }^{8,9}$, we also evaluated the discrepancy in $\cdot \mathrm{OH}$ level in mitochondria. For verification, we labelled mitochondria with MitoTracker® Red (red color) and evaluated beaconing lipid peroxidation with a Liperfluo probe. As shown in Fig. $2 \mathrm{G}$, the evolution trend of mitochondria lipid peroxidation level in various cases displayed similar characteristic to that demonstrated in the results regarding $\cdot \mathrm{OH}$ production illustrated in Fig.2F, with the FMUP-L group again showing the most lipid peroxidation in the mitochondria amongst all of the groups. Note that the addition of Lip-1, serving as a ROS scavenger ${ }^{37,38}$, alleviated lipid peroxidation in FMUP-L group, again indicating the role of $\cdot \mathrm{OH}$ production in the cell oxidative stress.

\section{Synergistic reinforcement of mitochondrial damage}

Having demonstrated the dual effects in terms of calcium overload and $\cdot \mathrm{OH}$ production in mitochondria, we moved on to investigate their synergistic outcomes on mitochondrial damage. To provide intuitional evidence, we started the investigation with TEM to reveal the change in morphology of HeLa cells after different treatments (Fig. 3A). It was found that cells after treatment of inert MU displayed typical mitochondrial morphology similar to that of cells in PBS group. In sharp contrast, other formulations of treatment with the involvement of the abovementioned enabling factors unequivocally gave rise to distinguishable morphological changes to the mitochondrial microstructure including profound swelling, outer membrane rupture and crista dissolution with the extent of mitochondria deconstruction augmented in the sequence of MU-L, FMU-L, and FMUP-L.

Considering that mitochondrial membrane potential (MMP) is typically sensitive to both calcium overload and the accumulation of ROS in mitochondria ${ }^{25,39}$, we next evaluated the MMP of HeLa cells after different treatments by using JC-1 as an indicator, which typically forms aggregates on normal mitochondrial membranes with a relatively high MMP while residing as monomers on the abnormal mitochondrial membrane with a low MMP. According to the flow cytometry data shown in Fig. 3B and D, the FMUP-L group showed the lowest proportion of aggregates $(28.8 \%)$ and the highest proportion of monomers $(70.7 \%)$ compared to the other groups, indicating the abnormality of MMP.

In addition to MMP, mitochondrial abnormalities can also be verified by the translocation of the cyclophilin $\mathrm{D}$ (Cyp D) protein from the cytoplasm to mitochondria, which is associated with the formation of the mitochondrial permeability transition pore complex ${ }^{40,41}$. To this end, we labelled mitochondria with MitoTracker ${ }^{\circledR}$ Green (green color) and evaluated the translocation of immunostaining Cyp D (red color). As expected, most of the Cyp D molecules had transferred to the mitochondria (Fig. 3C), and their colocalization rate was up to $76.71 \%$ (Fig. 3E) in the FMUP-L group, which was substantially higher than that of the other groups. Given that mitochondria serve as powerhouses in eukaryotic cells, we also detected ATP production to examine mitochondrial function. Compared with the PBS and MU groups, 
ATP productivity gradually decreased in the other advanced treatments, with the value decreasing to 0.1 in the FMUP-L group (Fig. 3F). Beyond doubt, these results unequivocally confirmed the most potent mitochondrial damage was induced by FMUP-L, which was ascribed to not only the improved uptake but also the synergism of calcium overload and ROS production.

\section{In vitro evaluation of cytotoxicity}

Encouraged by the abovementioned results regarding mitochondrial damage, we subsequently investigated the viability of HeLa cells in vitro after different treatments (Fig. 4A). All formulations exhibited a dose-dependent effect on the viability of HeLa cells, and the cytotoxicity increased in the following sequence: MU, MU-L, FMU-L, and FMUP-L. Taking the dose of $100 \mu \mathrm{g} / \mathrm{mL}$ as an example, less than $10 \%$ of cells survived in the FMUP-L group, while the cell viabilities in the other groups ranged from $\sim 40 \%$ to $~ 90 \%$. Given that two pathways were involved in mitochondrial damage, the possibility of ferroptosis induced by ROS production and apoptosis induced by calcium overload thus coexisted. To this end, we next investigated the two aspects of ferroptosis and apoptosis.

Ferroptosis was verified by the expression level of glutathione peroxidase 4 (GPX4), which has been regarded as the major indicator of oxidative stress due to its reduction capacity to reactive oxygen species (ROS) ${ }^{42,43}$. As the western blot data show in Fig. 4B, cells in MU group displayed GPX4 level roughly similar to the counterpart in PBS group due to the inert activity of MU in cells. Upon NIR lightassisted reduction of $\mathrm{Fe}^{3+}$ to $\mathrm{Fe}^{2+}$, the improved Fenton reaction and $\cdot \mathrm{OH}$ production definitely decreased the expression level of GPX4 (MU-L group) while the increase in internalization of nanoagents resulted in further suppression of GPX4 expression (FMU-L group). Particularly noteworthy was the involvement of pHP capable of photoacidification that significantly improved the efficiency of Fenton reaction and enabled highly efficient production of $\cdot \mathrm{OH}$ (FMUP-L group), which substantially inhibited the expression of GPX4 and consequently presented GPX4 level merely one-tenth of that in the PBS group. Subsequently, the level of intracellular caspase-3, a typical indicator of apoptosis ${ }^{44}$, was evaluated via immunostaining strategy with the results illustrated in Fig. 4C. It can be clearly seen that cells in the FMUP-L group displayed much higher level of caspase-3 as compared to the counterpart level in other groups, indicating the significant involvement of apoptosis in the former that was assigned to the photoacidification effect. Such cooperation of ferroptosis and apoptosis for cytotoxicity in the FMUP-L group was also verified by a live/dead assay (Fig. 4D). Compared with the other treatments, dead cells (red color) dominated the cell population in the FMUP-L group, indicating that most cells had been killed due to the combination of ferroptosis and apoptosis. Similar cytotoxic results were also observed in human liver carcinoma cells (HepG2) and mouse breast cancer cells (4T1) in Fig. 4E, and FMUP-L still substantially outperformed the other counterparts

\section{In vivo evaluation of biodistribution}

It is well documented that the ultimate therapeutic efficacy of anticancer nanomedicines critically depends on their in vivo fate. Taking this into account and being inspired by the aforementioned results, 
we assessed the fate of the engineered nanoagents in vivo. Considering that the FA ligand is highly correlated with targeting capacity, we enlisted FMUP and MUP for comparison, which were labelled by loading Cy7 dye into the MOF cavity. As the in vivo imaging data in Fig. 5A show, after intravenous injection, the majority of the MUP signals were rapidly enriched in the liver, with a minority of the MUP signals accumulating in the tumor site. In contrast, the fluorescence signal of FMUP at the tumor site increased substantially at the expense of the signal in the liver over time, indicating the pivotal role of doped FA for tumor accumulation. Despite this discrepancy, the accumulation of these two types of nanoagents at the tumor site peaked at $8 \mathrm{~h}$ (Fig. 5B). Upon further calculation of the signal ratio of the tumor/liver, we observed a magnified discrepancy evolution. Specifically, the ratio in the FMUP group increased and reached a peak value of $\sim 1.5$, while that in the MUP group continuously declined and reached 0.2 in $48 \mathrm{~h}$ (Fig. 5C).

To gain deeper insight, we excised the main organs, detected the fluorescence, and quantified the signal intensity. As shown in Fig. 5D and E, the fluorescence intensity of the excised tumor in the FMUP group was 2.4-fold higher than that in the MUP group, unequivocally proclaiming the FA-enabled tumortargeting capacity of FMUP for improved therapeutic performance. In other organs, the main discrepancy was found in the liver, and the FMUP group exhibited half of the accumulation the MUP group displayed, thus reducing the possibility of side effects to the liver. For higher resolution, these organs were further treated as frozen slices for microscopic observation (Fig. 5F). Compared with the MUP group, the FMUP group had substantially improved infiltration in the tumors and significantly suppressed accumulation in the liver. In addition to fluorescence-based measurements, the $\mathrm{T}_{2}$ signal of $\mathrm{Fe}^{3+}$ from our nanoagents also enabled us to investigate the biodistribution via MRI (Fig. 5G). FMUP displayed remarkably stronger contrast than MUP in $\mathrm{T}_{2}$ imaging at the tumor sites, again confirming its superior tumor targeting capacity.

\section{In vivo antitumor effects in cell derived xenograft (CDX) model}

The abovementioned in vivo results presented motivation for further investigation on the in vivo therapeutic performance of our nanoagents in HeLa tumor-bearing mice. For comparison, the HeLa tumor-bearing mice were randomly divided into five groups and treated with the formulation of PBS, MU, MU-L, FMU-L, and FMUP-L, respectively. In a typical protocol, mice in the groups with the involvement of light irradiation underwent irradiation of $980-\mathrm{nm}$ laser $\left(1.0 \mathrm{~W} / \mathrm{cm}^{2}, 5 \mathrm{~min}\right)$ twice, at $8 \mathrm{~h}$ and $32 \mathrm{~h}$, respectively, after a single intravenous administration (Fig. 6A). As shown in Fig. 6B, MU alone did not exert an appreciable influence on the growth of HeLa cells compared to the PBS group. Similar to the results observed in in vitro cytotoxicity (Fig. 4A), the factors of light irradiation, FA-enabled tumor targeting, and photoacid generator unequivocally played their respective roles in inhibiting the tumors with the consequent tumor size diminished in the sequence of MU-L, FMU-L, and FMUP-L. Specifically, treating tumor-bearing mice with FMUP-L nearly completely inhibited tumor growth with a $100 \%$ survival rate after 60 days (Fig. $6 \mathrm{C}$ ), which could be attributed to efficient tumor targeting as well as the combined effects of CDT and calcium overload-mediated mitochondrial damage. In contrast, all mice in the other groups gradually died within 50 days. 
Taking the synergistically reinforced mitochondrial damage for in vitro cytotoxicity, we evaluated whether this aspect was highly correlated with the above distinct therapeutic outcomes. To estimate the ability of regulating the intratumoral acidity, $\mathrm{SNARF}^{\circledR}-1$ was used as a probe and administered via intravenous tail injection to map $\mathrm{pH}$ fluctuations at the tumor sites on day 14 using an animal fluorescence imaging system (Fig. 6D). Referenced by the calibration curves obtained via the imaging system, the $\mathrm{pH}$ value at the tumor site after FMUP-L treatment was found decreased to 5.6, while the counterparts in other groups remained at $\sim 6.6$, highlighting the pivotal role of the photoacid generator in intratumoral acidification. Consequently, the intratumoral $\mathrm{Ca}^{2+}$ level (detected by the Fluo-3 probe) in the FMUP-L group was significantly higher than that in the other groups without the involvement of a photoacid generator (Fig. 6E), laying the foundation for FMUP-L treatment to enable calcium overload in tumor cells.

To verify the promoting effect of intratumoral acidification on Fenton reaction, we evaluated the ROS production at the tumor site via two-photon fluorescence microscopy using DCFH-DA as a probe. With the involvement of other promoting factors including the NIR-assisted reduction of $\mathrm{Fe}^{3+}$ to $\mathrm{Fe}^{2+}$ and the FAmediated improvement in internalization, the ROS productivity was found increased in the sequence of MU, MU-L, FMU-L, and FMUP-L (Fig. 6F). Due to the synergism of the above calcium overload and ROS production, the expression level of uncoupling protein 2 (UCP 2, a typical indicator of mitochondrial dysfunction ${ }^{45,46}$ ) in the FMUP-L group was markedly stronger than that in the other groups (Fig. 6G), indicating the most substantial mitochondrial damage in tumor cells in vivo. The FMUP-L group also displayed the fewest tumor cell nuclei in the H\&E slice (Fig. 6H). It also deserves mentioning that the potent antitumor effects of FMUP-L were achieved with few abnormalities to the histology of the main organs (Fig. S8), serum biochemical indicators (Fig. S9), and body weight (Fig. S10), indicating the good safety of our nanoagent.

\section{In vivo antitumor effects in patient derived tumor xenograft (PDX) model}

Cell lines widely used in routine antitumor experiments typically suffer from the lack of strong clinical relevance. As practical alternative models, PDX models mostly retain the principal histological and genetic characteristics of their donor tumor and remain stable across passages, which make them more reliable for clinical outcome prediction and preclinical drug evaluation. To confirm the clinical applicability of our nanoagent, we evaluated its therapeutic effect in a PDX model, which was established by transplanting a primary tumor sample resected from a liver cancer patient into the axilla of NOD-SCID mice followed by engraftment three times for subsequent use (Fig. 7A). Based on the immunostaining and flow cytometry analysis of dissociated cells, we confirmed that the high expression of the FA receptor remained in the PDX model (Fig. 7B). Additionally, FMUP (labelled by Cy7) showed 2.4 -fold accumulation in tumors (Fig. 7C) compared with its counterpart without FA functionalization. This FAmediated targeting capacity was further echoed at the histological level, with far more nanoagents observed in the tumor slice in the FMUP group than those in MUP group (Fig. 7D).

Having confirmed the targeting capacity of FMUP in the PDX model, we continued to investigate the therapeutic outcomes of FMUP-L treatment. To this end, patient-derived tumor-bearing mice were 
irradiated using a 980-nm laser $\left(1.0 \mathrm{~W} / \mathrm{cm}^{2}, 5 \mathrm{~min}\right)$ twice, at $8 \mathrm{~h}$ and $32 \mathrm{~h}$, respectively, after a single intravenous administration of FMUP nanoagent. Both in vivo imaging and two-photon microscopy imaging of tumors with pretreatment of $\mathrm{SNARF}^{\circledR}{ }_{-1} 1$ probe unequivocally confirmed that the $\mathrm{pH}$ value at the tumor site in the FMUP-L group was lower than that of the FMUP group (Fig. 7E and F). Downstream of intratumoral acidification was further evaluated by flow cytometry and frozen section staining imaging. Specifically, the FMUP-L group showed substantially shifted beaconing signal of DCFH-DA probe (Fig. 7G) and stronger signal of Fluo-3 (Fig. 7H) as compared to those in FMUP group, therefore strengthening the argument that intratumoral acidification could induce both calcium overload and efficient ROS production. Owing to the combination of these two enabling factors, significantly elevated expression of UCP 2 was observed on FMUP-L-treated tumor slices (Fig. 7I). Such potent mitochondrial damage resulted in prominent suppression of tumor proliferation (Fig. $7 \mathrm{~J}$ ) and almost complete inhibition of tumor growth (Fig. 7K). Correspondingly, the FMUP-L group showed a 100\% survival rate after 60 days, compared with all untreated mice that died within 25 days. These results together suggested that our FMUP-L nanoagent offered specific and highly effective antitumor therapy in the PDX model, thus shedding light on its potential clinical efficacy.

\section{Discussion}

The salient features of our FMUP nanoagent can be summarized in terms of the ingenious structureactivity relationships. The FMUP nanoagent is characterized with unique core-shell structures with UCNP as the core and MOF as the shell while photoacid generator component incorporated in the cavities of the MOF units. The UCNP core capable of upconverting NIR to UV light markedly circumvents the inherent impediments that UV light typically encounters in biological applications regarding phototoxicity to normal tissues on the optical path and the restricted penetration depth. Moreover, full exploitation of central metal ions, FA trims, and cavities in the MOF shell for antitumor action was realized for the Fenton reaction, targeting capacity, and pHP loading, respectively.

On the basis of the abovementioned design rationale, we succeeded in implementing synergistic damage to mitochondria with FMUP nanoagent. Specifically, 980-nm light mediated reduction of $\mathrm{Fe}^{3+}$ to $\mathrm{Fe}^{2+}$ and simultaneously activated the photoacid generator for acidifying the intracellular microenvironment.

Together with the released $\mathrm{Fe}^{2+}$, the acidic intracellular microenvironment, on the one hand, improved the efficiency of Fenton reactions for production of lethal $\cdot \mathrm{OH}$. Of particular note is that, as abovementioned, the upregulated $\mathrm{H}_{2} \mathrm{O}_{2}$ in mitochondria of tumor cells confined the $\cdot \mathrm{OH}$-based oxidative stress within the close proximity of mitochondria. On the other hand, intracellular acidification gave rise to calcium overload in mitochondria. The combination of the FA-enabled tumor targeting ability and the targetspecific damage based on the abovementioned two mechanisms tactfully confined the range of antitumor action locally to the tumors and therefore presented the desired antitumor specificity and efficacy. Based on the above synergistic damage to mitochondria, the FMUP nanoagent unequivocally displayed a potent killing effect on various tumor cell lines, including HeLa, 4T1, and HepG2. The in vivo experiments also provided unequivocal evidence that a single administration of FMUP with twice 
irradiation of 980-nm light could completely suppress tumor development in both CDX and PDX models, supporting FMUP-L as a promising modality to fight against cancer.

To the best of our knowledge, this is the first paradigm where a photoacid generator incorporated in MOF nanostructure was used for triggering synergistic damage to mitochondria based on the intratumoral photoacidification process and the consequent oxidative stress and calcium overload. This proof-ofconcept implementation opens up a way of exploring optically manipulating intracellular acidity/alkalinity for combined/synergistic antitumor therapeutics. For instance, replacing photoacid with photoalkali components (e.g., malachite green) and exploring the potential effects on cell viability and synergism with other therapeutic modalities could plausibly provide promising opportunities to antitumor applications. Further opportunities also exist in imparting additional and more potent therapeutic

activities via the way of optimizing the functional components of MOFs. For example, replacing $\mathrm{Fe}^{3+}$ with $\mathrm{Cu}^{2+}$ in constructing the MOF shells is expected to enable $\mathrm{Cu}^{+}$-mediated pyroptosis pathway and chemodynamic therapy based on the photocatalyzed reduction of $\mathrm{Cu}^{2+}$ to highly active $\mathrm{Cu}^{+}$for Fentonlike reaction. Additionally, integrating drug active ingredients into the organic linkers could be a way for introducing additional antitumor activity for further enhancement of therapeutic effects.

\section{Materials And Methods}

Materials. Folic acid (FA, 99.5\%), 1, 3, 5-benzene tricarboxylic acid (BTC, 99.5\%), $\mathrm{FeCl}_{3} \cdot 6 \mathrm{H}_{2} \mathrm{O}$, methylene blue (MB, 99.5\%) and methanol (99\%) were purchased from Sigma-Aldrich (U.S.A.). Penicillinstreptomycin, Dulbecco's modified eagle medium (DMEM), phosphate-buffered saline (PBS, pH = 7.4), and fetal bovine serum (FBS) were purchased from GIBCO BRL (Gaithersburg, MD, U.S.A.). Goat antirabbit IgG-Alexa 647, CCK-8, 2', 7'-Dichlorofluorescin diacetate (DCFH-DA), 5,5',6,6'-Tetrachloro-1,1',3,3'tetraethyl-imidacarbocyanine iodide (JC-1), and Hoechst 33342 were purchased from Beijing Solarbio Science\&Technology Co., Ltd. (Beijing, China). Anti-cyclophilin 40 (Cyp D) antibody, anti-mitochondrial calcium uniporter (MCU) antibody, anti-uncoupling protein 2 (UCP 2) antibody and anti-Ki 67 antibody were purchased from Abcam (Cambridge, UK). The liproxstatin-1 was purchased from Selleck Chemicals,

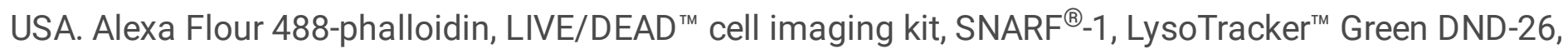
MitoTracker $^{\text {TM }}$ Green, MitoTracker ${ }^{\text {TM }}$ Red, and Fluo-3 were purchased from Life technologies, USA. The fluorescent hydrophilic dyes Cy5 and Cy7 were purchased from Fanbo Biochemical Co., Ltd. (Beijing, China). Liperfluo was purchased from Dojindo Molecular Technology, Inc. The upconversion (NaYF4: $\mathrm{Yb} / \mathrm{Tm}$, ultraviolet light emission, UCNP) was purchased from Suzhou Yansheng bio-tech Co., Ltd. Breast cancer line (4T1), human cervical carcinoma (HeLa), and liver hepatocellular cells (HepG 2) were purchased from Beijing Xiehe Hospital. Balb/c-nu mice were obtained from Vital River Laboratories (Beijing, China) and six-week-old NTG mice were purchased from SPF (Beijing) Biotechnology Co., Ltd. $980 \mathrm{~nm}$ laser meter (LWIRL980-10W-F, Beijing) was obtained from Beijing Laserwave Optoelectronics Technology Co., Ltd. (Beijing, China). The intensity of NIR laser used in the all experiments were 1.0 $\mathrm{W} / \mathrm{cm}^{2}$ and the irradiation time was $5 \mathrm{~min}$ for avoiding to generate heat in tissue. 
Preparation of photoacid. 4-(2-(4-hydroxy-3, 5-dimethoxyphenyl)-2-oxoethoxy)-4-oxobutan-1 -aminium was synthesized by the method reported in the literature. Typically, a solution of $\mathrm{Y}-0-(3,5-$ dimethoxy-4hydroxyphenacyl) t-butyl N-t-boc $\gamma$-aminobutyric acid (723 mg, $1.45 \mathrm{mmol}$ ) in $10 \mathrm{ml}$ trifluoroacetic acid (TFA) was cooled to $0{ }^{\circ} \mathrm{C}$ and reacted for $4 \mathrm{~h}$ with stirring. The resulting solution was concentrated by a rotary evaporator and residual solvent removal with a high vacuum pump. The crude product was extracted with a solution of $\mathrm{H}_{2} \mathrm{O} / \mathrm{EA}$. The aqueous layer was collected and the water was lyophilized off to give a clear oil of $\mathrm{y}$ - 0-(3,5-dimethoxy-4-hydroxyphenacyl) $y$-aminobutyric acid, trifluoroacetate salt (650 mg, 98\%).

Preparation of FMUP. FMUP were prepared using the method reported in the literature with minor modifications. In a typical procedure: first, $40 \mathrm{~mL}$ of $7.5 \mathrm{mM} \mathrm{FeCl}_{3}$ solution (in methanol) and $1.5 \mathrm{~mL}$ of UCNP methanol solution $(1 \mathrm{mg} / \mathrm{mL}$ ) were mixed under medium stirring for $30 \mathrm{~min}$. Afterward, $20 \mathrm{~mL}$ of $7.5 \mathrm{mM}$ BTC solution (in methanol), $20 \mathrm{~mL}$ of $7.5 \mathrm{mM}$ FA solution (in methanol), and $20 \mathrm{mg}$ photoacid pHP were added and mixed with the former solution for stirring about another $30 \mathrm{~min}$.

Next, the mixture was transferred into a $50^{\circ} \mathrm{C}$ Oil bath and reacted for 6 hours. The reaction product was collected by centrifugation (10000 rpm, $10 \mathrm{~min}$ ) and washed 3 times with ethanol and deionized water. Finally, the product was stored for use after drying. To facilitate the subsequent fluorescent imaging and measurement, The FMUP/Cy 5 or FMUP/Cy 7 could be obtained by mixing Cy 5 or Cy 7 into the reaction solution in the dark. The unloaded free Cy5 or Cy 7 molecules were removed by centrifugation at 10000 rpm with 10 min (DHS MC22R, China).

DLS characterization of FMUP. The size and zeta potential of FMUP or MUP were determined using a Malvern laser particle size analyzer; (NANO ZS, England). In order to determine stability of FMUP, the FMUP were stored in cell culture medium without fetal bovine serum (FBS). Then its size and zeta potential were detected every day.

TEM characterization of FMUP. The FMUP was dropped on the copper grid for three replicates. The morphology of the FMUP was observed by TEM at 100 kV (JEOL JEM-1400, Japan). In order to analyze the elements proportion of component in FMUP, a mapping scan analysis was performed.

BET characterization for different formulations. The Brunauer-Emmett-Teller (BET) surface area and pore size of FMU were measured using ASAP2050 system (England).

MRI characterization of FMUP. MRI of the FMUP solutions with various Fe concentrations was performed using a small animal MRI (9.4 T) scanner (Bruker, Germany) for three replicates.

Characterizations of FMUP before and after NIR light irradiation. The morphological changes of FMUP before and after NIR irradiation ( $980 \mathrm{~nm}, 1.0 \mathrm{~W} / \mathrm{cm}^{2}, 5 \mathrm{~min}$ ) were measured by TEM (JEOL JEM-1400, Japan). Meanwhile, DLS was used to determine the size of MOF after NIR irradiation. Correspondingly, we measured the amount of $\mathrm{Fe}^{2+}$ sourced from the FMUP. The concentration of $\mathrm{Fe}^{2+}$ and $\mathrm{Fe}^{3+}$ ions in the FMUP during NIR irradiation $\left(980 \mathrm{~nm}, 1.0 \mathrm{~W} / \mathrm{cm}^{2}\right.$ ) was determined by the standard Fe-detected method. 
$\mathrm{Fe}^{2+}$ can form a stable complex with 1,10-phenanthroline which has a maximum absorbance at $510 \mathrm{~nm}$. The absorbance of the complex was monitored using automatic microplate reader (Tecan Infinite M200, Switzerland) at $25^{\circ} \mathrm{C}$.

NIR light-triggered $\mathrm{pH}$ reversal in vitro. Before measuring the $\mathrm{pH}$ value change of solution, the standard solutions with different $\mathrm{pH}$ values $\left(5.0,5.5,6.0,6.5,7.0\right.$, and 7.5) were mixed with SNARF ${ }^{\circledR}-1$ at a volume ratio of 1:100, respectively. And the fluorescence intensities at $580 \mathrm{~nm}$ and $640 \mathrm{~nm}$ of various standard solutions were determined by automatic microplate reader. As a result, the calibration curve was obtained. The solution of FMUP mixed with SNARF $^{\circledR}-1$ at a volume ratio of 1:100 before NIR irradiation. Then, the mixed solution was irradiated continuously for $60 \mathrm{~min}\left(980 \mathrm{~nm}, 1.0 \mathrm{~W} / \mathrm{cm}^{2}\right)$. And fluorescence intensity at $580 \mathrm{~nm}$ and $640 \mathrm{~nm}$ of the FMUP solution were monitored by automatic microplate reader at room temperature every $5 \mathrm{~min}$. Then, the $\mathrm{pH}$ values with different irradiation time were calculated by the calibration curve.

Effect of NIR light-triggered acidic environment on ROS production in vitro. Before measuring generation of ROS in HeLa cells, the ability of generating more $\cdot \mathrm{OH}$ in acidic environment was evaluated and compared by MB bleaching assay. $200 \mu \mathrm{L}$ mixed solution comprising with MB (10 mg/L) and different formulations (FMUP, FMU-L, and FMUP-L) with equal Fe concentration reacted with $\mathrm{H}_{2} \mathrm{O}_{2}(200 \mu \mathrm{M})$ in 96well plate and further were irradiated with various time $(0 \mathrm{~min}, 1 \mathrm{~min}, 5 \mathrm{~min}, 10 \mathrm{~min}, 20 \mathrm{~min}, 30 \mathrm{~min}, 40$ $\mathrm{min}, 50 \mathrm{~min}$ and $60 \mathrm{~min}$ ), respectively. The absorption intensity at $664 \mathrm{~nm}$ at each time point was measured for determining the ability of the ROS production. Bleaching of $\mathrm{MB}$, due to the presence of $\cdot \mathrm{OH}$ in a sample, was indicated by a discoloration from a dark blue color to an almost white color. In addition, the $\cdot \mathrm{OH}$ production with various treatments were measured by electron spin-resonance spectroscopy (ESR). In detail, the reaction solution was mixed with 5,5-dimethyl-1-pyrroline N-oxide (DMPO) at a volume ratio of 1:50 before measurement.

The evaluation of FA on internalization by CLSM. For the cellular uptake study, HeLa cells were incubated with different formulations (FMUP/Cy 5 and MUP/Cy5). First, HeLa cells were seeded onto $35 \mathrm{~mm}$ glassbottom dishes at a density of $1 \times 10^{5}$ cells in $1 \mathrm{~mL}$ of culture media for adhesion. After $12 \mathrm{~h}$ adhesion, 50 $\mu \mathrm{L}$ Cy5 labeled various formulations $(1 \mathrm{mg} / \mathrm{mL})$ were added into $1 \mathrm{~mL}$ cell culture media. After $12 \mathrm{~h}$ internalization, the cells were washed by cold PBS, and then were fixed by $4 \%$ paraformaldehyde for 20 min. Next, the cell membrane and nuclei were stained with Alexa Fluor 488-phalloidin (Green) for 30 min in $37^{\circ} \mathrm{C}$ and Hoechst 33342 (Blue), respectively. The corresponding fluorescence images were obtained by CLSM (NIKON, A1).

The evaluation of FA on internalization by Flow cytometry. Flow cytometry (Beckman Coulter CyAn ADP, USA) was applied to further quantify the cellular uptake in different groups (FMUP/Cy 5 and MUP/Cy5). First, HeLa cells were seeded in 48-well plate for $12 \mathrm{~h}$ until cell attached the bottom. Next, the cells were incubated with $0.05 \mathrm{mg} / \mathrm{mL}$ Cy 5 labeled MOFs for another $12 \mathrm{~h}$. Subsequently, cells were washed by cold PBS with three times and further were collected by centrifugation (1000 rpm, 5min). Finally, the uptake amount upon different treatments was determined by flow cytometer (FCM). Data were obtained from 
15000 cells for each sample. In order to confirm the effect of endocytosis time on the amount of endocytosis, the same method was executed within different internalized times.

Effect of NIR light-triggered acidic environment on escaping lysosome by CLSM. The cultural conditions for HeLa cells were described above and incubated with $0.05 \mathrm{mg} / \mathrm{mL}$ Cy 5 labeled FMUP for $12 \mathrm{~h}$ incubation. Before labeling lysosome, the cells were irradiated by NIR with $5 \min \left(980 \mathrm{~nm}, 1.0 \mathrm{~W} / \mathrm{cm}^{2}\right)$, and were put into incubator for $1 \mathrm{~h}$. For comparison, the cells treated with FUMP were not suffered from NIR irradiation. Next, Lyso-Tracker Green at a concentration of $50 \mathrm{nM}$ was added and incubated with cells for $30 \mathrm{~min}$ at $37^{\circ} \mathrm{C}$. Before observation, the unreacted dye was removed and the cells were washed by cold PBS. Lyso-Tracker Green was excited at $488 \mathrm{~nm}$ and Cy 5 in FMUP/Cy5 was excited at $664 \mathrm{~nm}$, respectively. The corresponding fluorescent images at 500-545 nm and 660-710 nm were obtained by CLSM. And the co-localization rates of FMUP and lysosomes in various treatments were calculated by NIKON analyzer.

Effect of NIR light-triggered acidic environment on escaping lysosome by TEM. Different groups' cell was cultured and treated with the described condition. And different treated cells were fixed with $1 \mathrm{~mL}$ general fixative (containing $2.5 \%$ glutaraldehyde in $0.1 \mathrm{M}$ Phosphate buffer) at $4{ }^{\circ} \mathrm{C}$ for overnight. After dehydration, cells were embedded in epoxy resin and the resin was stored at $55^{\circ} \mathrm{C}$ for $48 \mathrm{~h}$ to allow resin polymerization. The embedded samples were then sliced with a thickness of 50-70 nm. Finally, the cell sections were stained with $5 \%$ uranyl acetate for $15 \mathrm{~min}$ and $2 \%$ lead citrate for $15 \mathrm{~min}$ before TEM imaging.

Assessment of NIR light-triggered acidification of HeLa cells by CLSM. The cultural conditions for HeLa cells were described above and FMUP were added and incubated into the cells at $0.05 \mathrm{mg} / \mathrm{mL}$ for $12 \mathrm{~h}$. Before NIR irradiation $\left(980 \mathrm{~nm}, 1.0 \mathrm{~W} / \mathrm{cm}^{2}, 5 \mathrm{~min}\right.$ ), the cells were incubated with $\mathrm{pH}$ indicator (SNARF $\left.{ }^{\circledR}-1\right)$ at $50 \mathrm{nM}$ for $30 \mathrm{~min}$. SNARF ${ }^{\circledR}-1$ was excited at $488 \mathrm{~nm}$. The corresponding fluorescent images at 560 $590 \mathrm{~nm}$ and $620-650 \mathrm{~nm}$ were taken by CLSM. And the fluorescence ratio of intensity $580 \mathrm{~nm}$ and intensity $640 \mathrm{~nm}$ were calculated by NIKON analyzer. Subsequently, the $\mathrm{pH}$ value was calculated by using the calibration curve.

In vitro assessment of NIR light-triggered acidification on calcium influx by CLSM. The cultural conditions for HeLa cells were described above and FMUP or FMU were added at $0.05 \mathrm{mg} / \mathrm{mL}$ for $12 \mathrm{~h}$ incubation. Before NIR irradiation, the cells were incubated with $\mathrm{Ca}^{2+}$ indicator (Fluo-3) at $5 \mu \mathrm{M}$ for $30 \mathrm{~min}$. Then, the excess Fluo-3 and MOFs were removed and washed by cold PBS in dish. Finally, the fresh DMEM media were added into the dish. Next, we employed the CLSM to monitor the distribution of $\mathrm{Ca}^{2+}$ in $\mathrm{HeLa}$ cells (denoted as $-5 \mathrm{~min}$ ). Subsequently, the cells were received NIR irradiation $\left(980 \mathrm{~nm}, 1.0 \mathrm{~W} / \mathrm{cm}^{2}, 5 \mathrm{~min}\right)$ in each group (FMUP-L and FMU-L). Finally, we performed point-in-time imaging and calculated the concentration of calcium in cell with elapsed time.

In vitro assessment of NIR light-triggered acidification on calcium overload in mitochondria by CLSM. Different groups' cell was treated and cultured with the described condition in $35 \mathrm{~mm}$ glass-bottom 
dishes. After NIR irradiation ( $\left.980 \mathrm{~nm}, 1.0 \mathrm{~W} / \mathrm{cm}^{2}, 5 \mathrm{~min}\right)$, mitochondria were stained by MitoTracker ${ }^{\mathrm{TM}}$ Green at $200 \mathrm{nM}$ for $30 \mathrm{~min}$ in $37^{\circ} \mathrm{C}$. Then cells in different groups were washed by cold PBS and were fixed by $4 \%$ paraformaldehyde for $30 \mathrm{~min}$ in $37^{\circ} \mathrm{C}$. Subsequently, cells were incubated with $0.2 \%$ Triton X100 in PBS for 10 min to achieve good permeabilization. Immediately, the cells were blocked with blocking buffer for $2 \mathrm{~h}$ at room temperature. Primary antibody to the mitochondrial calcium uniporter (MCU) was incubated with the cells in $4^{\circ} \mathrm{C}$ overnight. After the cells were washed three times by cold PBS, they were incubated with goat anti-rabbit IgG-Alexa 647 secondary antibody for $2 \mathrm{~h}$ in room temperature. Red MCU was exited at $633 \mathrm{~nm}$. Green mitochondrial was exited at $488 \mathrm{~nm}$. The corresponding fluorescent images were obtained by CLSM. And the expression level of MCU was calculated by NIKON analyzer.

In vitro evaluation of NIR light-triggered acidification on ROS generation by CLSM. The cultural conditions for HeLa cells were described above, and different formulations were added at $0.05 \mathrm{mg} / \mathrm{mL}$ overnight. The ROS were reacted with DCFH-DA for $20 \mathrm{~min}$ in $37^{\circ} \mathrm{C}$ before NIR irradiation. Based on the Fenton reaction, $\mathrm{Fe}^{2+}$ could react with $\mathrm{H}_{2} \mathrm{O}_{2}$ to produce ROS more strongly and actively in acidic environment than that in weak alkaline environment after NIR irradiation $\left(980 \mathrm{~nm}, 1.0 \mathrm{~W} / \mathrm{cm}^{2}, 5 \mathrm{~min}\right)$. Then the cellular ROS oxidized DCF can be used as indicator for ROS production. DCF was excited at $488 \mathrm{~nm}$. The corresponding fluorescent images of cellular DCF at excitation wavelength of 510-555 nm were taken by CLSM.

In vitro evaluation of NIR light-triggered acidification on lipid peroxidation by CLSM. Different groups' cell was treated with the described condition. The lipid peroxidation and mitochondria in cells with different treatments were stained by lipid peroxidation probe (Liperfluo) and MitoTracker ${ }^{\mathrm{TM}}$ Red, respectively. In detail, the cells were incubated with lipid peroxidation probe (Liperfluo) at $10 \mu \mathrm{M}$ for $30 \mathrm{~min}$ in $37^{\circ} \mathrm{C}$ before NIR irradiation. Meanwhile, mitochondria were stained by MitoTracker ${ }^{\mathrm{TM}}$ Red at $200 \mathrm{nM}$ for 30 min in $37^{\circ} \mathrm{C}$. After irradiation, the corresponding fluorescent images from various treatments could be obtained by CLSM. And the co-localization rates of lipid peroxidation (green) and mitochondria (red) in various treatments were calculated by NIKON analyzer.

\section{In vitro evaluation of NIR light-triggered acidification on ferroptosis by simple western immunoblots. In} brief, HeLa cells were seeded in 48-well plates at a density of $1 \times 10^{6}$ cells per well for attaching. Then the cells were treated with different formulations (equivalent concentration $50 \mu \mathrm{g} / \mathrm{mL}$ ). The expressions of GPX4 proteins were evaluated by western blot for ferroptosis. Cytoplasm protein was extracted by Minute TM Cytoplasmic and Nuclear Extraction Kit (Invent Biotechnologies, USA). Protein concentration of each groups were measured by the BCA method. The protein was incubated with rabbit anti-mouse polyclonal antibody (1:1000, Santa Cruz, USA). The protein antibody complexes were detected using the HRP (Horseradish peroxidase) conjugated secondary antibody (1:5000) (Earthox, USA). Protein bands were visualized using automatic protein expression analysis system (Wes System, USA).

\section{In vitro evaluation of NIR light-triggered acidification on mitochondrial membrane potential by FCM. In} short, HeLa cells were seeded in 48-well plates at a density of $1 \times 10^{6}$ cells per well for attaching 
overnight. Then the wells were added to different formulations (equal concentration $50 \mu \mathrm{g} / \mathrm{mL}$ ) for incubating $12 \mathrm{~h}$. After NIR irradiation $\left(980 \mathrm{~nm}, 1.0 \mathrm{~W} / \mathrm{cm}^{2}, 5 \mathrm{~min}\right)$, the probe (JC-1) for measuring mitochondrial membrane potential $(10 \mu \mathrm{g} / \mathrm{mL})$ incubated with cells for $10 \mathrm{~min}$ in $37^{\circ} \mathrm{C}$. Next, the cells were washed by cold PBS with three times before collecting cells. And the mitochondrial membrane potential was determined on flow cytometer. The receiving emission wavelength of the JC-1 monomer is $529 \mathrm{~nm}$ and the receiving emission wavelength of the JC-1 aggregate is $590 \mathrm{~nm}$. Data were obtained from 15000 cells for each sample.

Effect of NIR light-triggered acidic environment on mitochondrial morphology by CLSM. Different groups' cells were treated with the described condition in $35 \mathrm{~mm}$ glass-bottom dishes. After NIR irradiation, mitochondria in cells were stained by MitoTracker ${ }^{\mathrm{rm}} \mathrm{Green}$ at $200 \mathrm{nM}$ for $30 \mathrm{~min}$ in $37^{\circ} \mathrm{C}$. Then cells of different groups were washed by cold PBS and were fixed by $4 \%$ paraformaldehyde for 30 min in $37^{\circ} \mathrm{C}$. Next, Cells were incubated with $0.2 \%$ Triton X-100 in PBS for 10 min to achieve good permeabilization. Immediately, the cells were blocked with blocking buffer for $2 \mathrm{~h}$ at room temperature. Primary antibody to the mitochondrial membrane pore-associated protein Cyp $\mathrm{D}$ was incubated with the cells at $4^{\circ} \mathrm{C}$ overnight. After the cells were washed three times by cold PBS, they were incubated with goat anti-rabbit IgG-Alexa 647 secondary antibody for $2 \mathrm{~h}$ at room temperature. Green mitochondrial was excited at 488 $\mathrm{nm}$. Red Cyp D was exited at $633 \mathrm{~nm}$. The corresponding fluorescent images of mitochondrial and Cyp D were taken by CLSM. And the co-localization of these images was calculated by NIKON analyzer.

Effect of NIR light-triggered acidic environment on mitochondrial morphology by TEM. Different groups' cell was treated with the described condition. Before TEM imaging, cells were fixed, dehydrated and stained in the above section. For detailed operation steps, please refer to the previous article.

Effect of NIR light-triggered acidic environment on mitochondrial function. For determining the function of mitochondria, the standard method was employed to measure the ATP production. In detail, HeLa were seeded in 96 -well plate at a density of $1.5 \times 10^{4}$ cells and cultured overnight for allowing attachment. Then the cells were incubated with different formulations (MU, MU-L, FMU-L, and FMUP-L) with equal concentration of $\mathrm{MU}(50 \mu \mathrm{g} / \mathrm{mL})$ for another $12 \mathrm{~h}$. Next, the cells were collected for detecting the ATP production. According to the kit instructions, the absorption intensity of each well at $340 \mathrm{~nm}$ was measured by automatic microplate reader. And the amount of ATP was calculated.

CCK-8 cytotoxicity assay for different formulations. The cytotoxicity was determined using the CCK-8 (Beyotime, China) assay. Briefly, HeLa, 4T1 cells or HepG 2 were seeded in 96-well plate at a density of $1.5 \times 10^{4}$ cells and cultured overnight for allowing cell attachment. Then the cells were incubated with different formulations (equivalent $\mathrm{MU}$ concentration ranged from 0 to $100 \mu \mathrm{g} / \mathrm{mL}$ ) for another $12 \mathrm{~h}$. CCK8 test solution was added to each well of the plate (The volume of the CCK-8 test solution in each well is one tenth of the total volume) and incubated for another $3 \mathrm{~h}$. The absorption intensity of each well at 450 $\mathrm{nm}$ was measured by automatic microplate reader. And the cell viability was calculated and normalized. 
Assessment of NIR light-triggered acidification on apoptosis by CLSM. Different groups' cell was treated with the described condition in $35 \mathrm{~mm}$ glass-bottom dishes. After NIR irradiation, nuclei were stained by Hoechst 33342 with 20 min in $37^{\circ} \mathrm{C}$. Then cells of different groups were washed by cold PBS and were fixed by $4 \%$ paraformaldehyde for $30 \mathrm{~min}$ in $37^{\circ} \mathrm{C}$. Subsequently, Cells were incubated with $0.2 \%$ Triton X100 in PBS for 10 min to achieve good permeabilization. Immediately, the cells were blocked with blocking buffer for $2 \mathrm{~h}$ at room temperature. Primary antibody to the caspase-3 was incubated with the cells at $4^{\circ} \mathrm{C}$ overnight. After the cells were washed three times by cold PBS, they were incubated with goat anti-rabbit IgG-Alexa 647 secondary antibody for $2 \mathrm{~h}$ at room temperature. Blue nuclei were excited at 405 $\mathrm{nm}$. Pink caspase-3 was exited at $633 \mathrm{~nm}$. The corresponding fluorescent images were taken by CLSM. And the expression level of caspase-3 was calculated by NIKON analyzer.

In vitro evaluation of cellular apoptosis by live/dead assay. HeLa cells were cultured as described above, then different MOFs formulations (equivalent MOF concentration $100 \mu \mathrm{g} / \mathrm{mL}$ and corresponding additives) were added for $12 \mathrm{~h}$. The cells were stained by Calcein-AM/EthD- 1 staining working solution for 20 min at $37^{\circ} \mathrm{C}$. Green $(494 / 517 \mathrm{~nm})$ viable cells can be stained by Calcein AM, while red (528/617 $\mathrm{nm})$ dead cells can be stained by EthD-1.

In vivo evaluation of tumor targeting. Balb/c-nu mice (4-6 weeks old) were bought from Vital River Laboratories (Beijing, China) and used for animal experiments directly. The tumors were obtained by injecting female mice with HeLa cells $\left(8.0 \times 10^{7}\right.$ cells in $100 \mu \mathrm{L}$ of PBS). In order to observe the biodistribution of different formulation in vivo, equivalent various MOFs (MUP/Cy7 or FMUP/Cy7) were intravenously injected to HeLa tumor-bearing mice and their biodistribution were observed by using animal imaging system (Kodak FX Pro, Japan) at different time point. Then the tumors and organs were excised and imaged after $48 \mathrm{~h}$. Due to MRI property of $\mathrm{Fe}^{3+}$, mice in different groups were imaged by MRI (BioSpec70/20USR, Germany) for tracking and diagnosis at tumor site before and after injection.

In vivo evaluation of anticancer efficacy in HeLa model. For investigating their antitumor effect of different MOF formulations, HeLa tumor-bearing mice were treated when the average tumor volume reached approximately $100 \mathrm{~mm}^{3}$. The mice were randomly divided into five groups (each group $\mathrm{n}=6$ ). Tumor volumes $(V)$ were measured for five groups of mice that were intravenously administered with PBS, MU, MU-L (980 nm, 1.0 W/cm², 5 min), FMU-L (980 nm, 1.0 W/cm², 5 min), and FMUP-L (980 nm, 1.0 $\mathrm{W} / \mathrm{cm}^{2}, 5 \mathrm{~min}$ ) until day 26 . Note that mice in the groups with the involvement of light irradiation underwent treatment of irradiation $\left(980 \mathrm{~nm}, 1.0 \mathrm{~W} / \mathrm{cm}^{2}, 5 \mathrm{~min}\right)$ twice, at $8 \mathrm{~h}$ and $32 \mathrm{~h}$, respectively, after a single intravenous administration. Tumor volumes were calculated by:

$$
V=\frac{L \times W^{2}}{2}
$$

where $L$ is the longest and $W$ is the shortest tumor diameter $(\mathrm{mm})$. 
Antitumor study in humanized PDX model. The PDX tumor samples were s.c. transplanted into the axilla of the NTG mice to establish the PDX model and then these mice were randomly divided into two groups: PBS group and FMUP+L group. Two weeks later, when the tumors were visible $\left(\sim 150 \mathrm{~mm}^{3}\right)$, the mice of these groups were i.v. injected with PBS and FMUP, respectively. Eight hour later, the mice in $\mathrm{FMUP}+\mathrm{L}$ group were anaesthetized and the tumors of them were illuminated with a $980-\mathrm{nm}$ laser $\left(1.0 \mathrm{~W} / \mathrm{cm}^{2}, 5\right.$ $\mathrm{min})$. Note that mice in the groups with the involvement of light irradiation underwent treatment of irradiation ( $980 \mathrm{~nm}, 1.0 \mathrm{~W} / \mathrm{cm}^{2}, 5 \mathrm{~min}$ ) twice, at $8 \mathrm{~h}$ and $32 \mathrm{~h}$, respectively, after a single intravenous administration. Finally, the tumor sizes and survival percent were measured every two days, and the experimental endpoint was defined as either death or the tumor size greater than $1500 \mathrm{~mm}^{3}$. The biodistribution study was administered in the same ways as we used in the corresponding studies. And the acidification study was investigated by the multiphoton laser confocal microscopy. The evaluation of ROS generation in tumor was measured by FCM. Besides, the investigation for calcium influx in tumor was detected by frozen section staining using Fluo-3. Moreover, the evaluation of mitochondria damage was measured via immunohistochemical staining. The haematoxylin and eosin (H\&E) staining of tumor sections for the study was administered in the same way. The study was approved by Shanghai Tongren Hospital medical ethics committee in accordance with the 1964 Declaration of Helsinki and 1982 International Ethical Guidelines for Human Biomedical Research (2019-032-01, 2020-059-01 and 2021003-01).

Evaluation of acidification on tumor by animal imaging system. Before measuring the $\mathrm{pH}$ values in tumor sites, the standard solutions with different $\mathrm{pH}$ values $(5.0,5.5,6.0,6.5,7.0$, and 7.5$)$ were mixed with $\mathrm{SNARF}^{\circledR}-1$ at a volume ratio of $1: 100$, respectively. And the fluorescence intensities at $580 \mathrm{~nm}$ and 640 $\mathrm{nm}$ of various standard solutions were determined by animal imaging system. As a result, the calibration curve was obtained. To estimate the $\mathrm{pH}$ reversal in HeLa the tumor site, the mice with different treatments (PBS, MU, MU-L, FMU-L, and FMUP-L) were intravenously injected with a pH-sensitive fluorescent dye (SNARF $\left.{ }^{\circledR}-1\right)$ at 20 min before imaging. After that, the mice received NIR irradiation in the corresponding groups. Finally, the $\mathrm{pH}$ values in tumor sites were calculated by the ratio of the fluorescence at $580 \mathrm{~nm}$ and $640 \mathrm{~nm}$.

Evaluation of acidification on ROS production in tumor. In order to investigate the generation of ROS in situ, DCFH-DA was intratumoral administered before imaging by multiphoton laser confocal scanning microscopy. And then mice's tumor was irradiated by NIR $\left(980 \mathrm{~nm}, 1.0 \mathrm{~W} / \mathrm{cm}^{2}, 5 \mathrm{~min}\right)$. Subsequently, the green ROS was detected and measured by multiphoton laser confocal scanning microscopy and further quantitative analysis for different treatments.

\section{Evaluation of the synergistic effect on mitochondria by immunohistochemical section. After the} completion administering, one mouse was taken from each group for immunofluorescence analysis. The expression of uncoupling protein 2 (UCP 2) in mitochondrial membrane induced by the synergistic effect was also verified by immunohistochemistry. 
Evaluation of the synergistic effect on tumor inhibition by immunohistochemical section. After the completion administering, one mouse was taken from each group for immunofluorescence analysis. Proliferating cell nuclear antigen- $\mathrm{K}_{\mathrm{i}} 67$ was used for determining nucleus proliferating of tumor tissue.

Safety evaluation of different treatments. For further investigate the safety of different formulations in vivo, the body weight of mice in each groups were recorded until 60 days. Besides, the serum levels of urea nitrogen (BUN), lactate dehydrogenase (LDH), alanine aminotransferase (ALT), aspartate transaminase (AST), and alkaline phosphatase (ALP) were analyzed by using an automated analyzer (Hitachi Ltd Hitachi-917, Japan). The main organs (heart, liver, spleen, lung, and kidney) were sliced and stained by hematoxylin-eosin (H\&E) staining.

Animal care. Balb/c-nu mice and NTG mice, 4-6 weeks of age, were obtained from Vital River laboratories (Beijing, China). This study was performed in strict accordance with the Regulations for the Care and Use of Laboratory Animals and Guideline for Ethical Review of Animal (China, GB/T 35892-2018). All animal experiments were reviewed and approved by the Animal Ethics Committee of the Institute of Process Engineering (approval ID: IPEAECA2019318).

Statistical analysis. All the data are presented as the mean \pm SD. Statistical analysis was performed with Prism 8.0 software (GraphPad Software) by an unpaired Student's t-test, log-rank test, one-way ANOVA.

\section{Declarations}

Reporting summary. Further information on research design is available in the Nature Research Reporting Summary linked to this article.

\section{Data availability}

The authors declare that all relevant data are included in the paper and supplementary information files. Source data are provided with this paper.

\section{Acknowledgements}

This work was supported by the National Natural Science Foundation of China (No. 22077121, 21773241, 32030062, 21821005, and U2001224), the National Key R\&D Program of China (2017YFA0207900), and National Science and Technology Major Project of China (2018ZX10301-1-03003).

\section{Author contributions}

Z. T., W. W. and G. M. designed and supervised the whole project. W. B., J. M., M. L., S. L., and S. W., performed the researches and experiments. Y. W. and R. J. contributed to the PDX model. W. B., Z. T., and W.W. collected and analyzed the data. W. B., Z. T., and W. W. wrote the initial manuscript. All the authors commented on the manuscript and approved the submission. 


\section{Competing interests}

The authors declare no competing financial interest.

\section{Additional information}

Supplementary information is available for this paper at http://

Correspondence and requests for materials should be addressed to G. M., W. W., or Z. T.

Peer review information Nature Communications thanks the anonymous reviewer(s) for their contribution to the peer review of this work. Peer reviewer reports are available.

Reprints and permissions information is available at www.nature.com/reprints.

Publisher's note: Springer Nature remains neutral with regard to jurisdictional claims in published maps and institutional affiliations.

\section{References}

1 Dantzer, R., Meagher, M. W. \& Cleeland, C. S. Translational approaches to treatment-induced symptoms in cancer patients. Nat. Rev. Clin. Oncol. 9, 414-426 (2012).

2 Cleeland, C. S. et al. Reducing the toxicity of cancer therapy: recognizing needs, taking action. Nat. Rev. Clin. Oncol. 9, 471-478 (2012).

3 Blumenfeld, C. M. et al. Drug capture materials based on genomic DNA-functionalized magnetic nanoparticles. Nat. Commun. 9, 2870 (2018).

4 Vasan, N., Baselga, J. \& Hyman, D. M. A view on drug resistance in cancer. Nature 575, 299-309, (2019).

5 Das, M., Alzaid, F. \& Bayry, J. Regulatory T Cells under the mercy of mitochondria. Cell Metab. 29, 243-245 (2019).

6 Giorgi, C., Danese, A., Missiroli, S., Patergnani, S. \& Pinton, P. Calcium dynamics as a machine for decoding signals. Trends Cell Biol. 28, 258-273 (2018).

7 Balaban, R. S., Nemoto, S. \& Finkel, T. Mitochondria, oxidants, and aging. Cel/ 120, 483-495 (2005).

8 Lee, S. et al. Mitochondrial $\mathrm{H} 2 \mathrm{O} 2$ generated from electron transport chain complex I stimulates muscle differentiation. Cell Res. 21, 817-834 (2011). 
9 Giorgio, M., Trinei, M., Migliaccio, E. \& Pelicci, P. G. Hydrogen peroxide: a metabolic by-product or a common mediator of ageing signals? Nat. Rev. Mol. Cell Biol. 8, 722a-728 (2007).

10 Tang, Z., Liu, Y., He, M. \& Bu, W. Chemodynamic Therapy: Tumour microenvironment-mediated Fenton and Fenton-like reactions. Angew. Chem., Int. Ed. Engl. 58, 946-956 (2019).

11 Tang, Z. M. et al. Antiferromagnetic pyrite as the tumor microenvironment-mediated nanoplatform for self-enhanced tumor imaging and therapy. Adv. Mater. 29, 1701683 (2017).

12 Fan, J. X. et al. Engineered bacterial bioreactor for tumor therapy via Fenton-like reaction with localized H2O2 generation. Adv. Mater. 31, 1808278 (2019).

13 Li, X. S., Kwon, N., Guo, T., Liu, Z. \& Yoon, J. Innovative strategies for hypoxic-tumor photodynamic therapy. Angew. Chem., Int. Engl. 57, 11522-11531 (2018).

14 Kang, Y. W. \& Hwang, K. Y. Effects of reaction conditions on the oxidation efficiency in the Fenton process. Water Res. 34, 2786-2790 (2000).

15 Webb, B. A., Chimenti, M., Jacobson, M. P. \& Barber, D. L. Dysregulated pH: a perfect storm for cancer progression. Nat. Rev. Cancer 11, 671-677 (2011).

16 Bao, W. et al. Nanolongan with multiple On-demand conversions for ferroptosis-apoptosis combined anticancer therapy. ACS Nano 13, 260-273 (2019).

17 Zhang, C. et al. Synthesis of iron nanometallic glasses and their application in cancer therapy by a localized Fenton reaction. Angew. Chem., Int. Engl. 55, 2101-2106 (2016).

18 Zhang, Y. et al. A near-infrared photo-switched microRNA amplifier for precise photodynamic therapy of early-stage cancers. Angew. Chem., Int. Engl. 59, 21454-21459 (2020).

19 Di, Z. H. et al. An orthogonally regulatable DNA nanodevice for spatiotemporally controlled biorecognition and tumor treatment. Sci. Adv. 6, eaba9381 (2020).

20 Peng, F., Li, G. Z., Liu, X. X., Wu, S. Z. \& Tong, Z. Redox-responsive gel-sol/sol-gel transition in poly(acrylic acid) aqueous solution containing Fe(III) ions switched by light. J. Am. Chem. Soc. 130, 16166-16171 (2008).

21 Graedel, T. E., Weschler, C. J. \& Mandich, M. L. Influence of transition-metal complexes on atmospheric droplet acidity. Nature 317, 240-242 (1985).

22 Zhu, X. et al. Photoreduction of iron(iii) in marine mineral aerosol solutions. J. Geophys. ResAtmos. 98, 9039-9046 (1993).

23 Pedersen, S. F., Jorgensen, N. K. \& Hoffmann, E. K. Dynamics of Ca-i(2+) and pH(i) in Ehrlich ascites tumor cells after $\mathrm{Ca} 2+-$ mobilizing agonists or exposure to hypertonic solution. Pflug. Arch. Eur. J. 
Phy. 436, 199-210 (1998).

24 Eskiocak, U. et al. Synergistic effects of ion transporter and MAP kinase pathway inhibitors in melanoma. Nat. Commun. 7, 13080 (2016).

25 Zheng P. et al. Ultrasound-augmented mitochondrial calcium ion overload by calcium nanomodulator to induce immunogenic cell death. Nano Lett. 21, 2088-2093 (2021).

$26 \mathrm{Xu}, \mathrm{L} . \mathrm{H}$. et al. Enhanced intracellular Ca2+ nanogenerator for tumor-specific synergistic therapy via disruption of mitochondrial Ca2+ homeostasis and photothermal therapy. ACS Nano 12, 6806-6818, (2018).

27 Burns, K. D., Homma, T., Breyer, M. D. \& Harris, R. C. Cytosolic acidification stimulates a calcium influx that activates $\mathrm{Na}+-\mathrm{H}+$ exchange in Llc-Pk1. Am. J. Physiol. 261, 617-625 (1991).

28 Conrad, P. G., Givens, R. S., Weber, J. F. W. \& Kandler, K. New Phototriggers: ${ }^{1}$ Extending the pHydroxyphenacyl $\pi-\pi^{\star}$ Absorption Range. Org. Lett. 2, 1545-1547 (2000).

29 Conrad, P. G. et al. p-hydroxyphenacyl phototriggers: the reactive excited state of phosphate photorelease. J. Am. Chem. Soc. 122, 9346-9347 (2000).

30 Sega, E. I. \& Low, P. S. Tumor detection using folate receptor-targeted imaging agents. Cancer Metastasis Rev. 27, 655-664 (2008).

31 Chen, L. et al. Tumor-targeted drug and $\mathrm{CpG}$ delivery system for phototherapy and docetaxelenhanced immunotherapy with polarization toward M1-type macrophages on triple negative breast cancers. Adv. Mater. 31, 1904997 (2019).

32 Lim, S. H. et al. In vitro and in vivo photocytotoxicity of boron dipyrromethene derivatives for photodynamic therapy. J. Med. Chem. 53, 2865-2874 (2010).

33 Hatz, S., Lambert, J. D. C. \& Ogilby, P. R. Measuring the lifetime of singlet oxygen in a single cell: addressing the issue of cell viability. Photoch. Photobio. Sci. 6, 1106-1116 (2007).

34 Kirichok, Y., Krapivinsky, G. \& Clapham, D. E. The mitochondrial calcium uniporter is a highly selective ion channel. Nature 427, 360-364 (2004).

35 Kamer, K. J. \& Mootha, V. K. The molecular era of the mitochondrial calcium uniporter. Nat. Rev. Mol. Cell Biol. 16, 545-553 (2015).

36 Rizzuto, R., De Stefani, D., Raffaello, A. \& Mammucari, C. Mitochondria as sensors and regulators of calcium signalling. Nat. Rev. Mol. Cell Biol. 13, 566-578 (2012). 
37 Zilka, 0. et al. On the mechanism of cytoprotection by ferrostatin-1 and liproxstatin-1 and the role of lipid peroxidation in ferroptotic cell death. ACS Cent. Sci. 3, 232-243 (2017).

38 Angeli, J. P. F. et al. Inactivation of the ferroptosis regulator Gpx4 triggers acute renal failure in mice. Nat. Cell Biol. 16, 1180-1120 (2014).

39 Van Loo, G. et al. The role of mitochondrial factors in apoptosis: a Russian roulette with more than one bullet. Cell Death Differ. 9, 1031-1042 (2002).

40 Halestrap, A. P., Woodfield, K. Y. \& Connern, C. P. Oxidative stress, thiol reagents, and membrane potential modulate the mitochondrial permeability transition by affecting nucleotide binding to the adenine nucleotide translocase. J. Biol. Chem. 272, 3346-3354 (1997).

41 Lin, D. T. \& Lechleiter, J. D. Mitochondrial targeted cyclophilin D protects cells from cell death by peptidyl prolyl isomerization. J. Biol. Chem. 277, 31134-31141 (2002).

42 Liang, C., Zhang, X. L., Yang, M. S. \& Dong, X. C. Recent progress in ferroptosis inducers for cancer therapy. Adv. Mater. 31, 1904197 (2019).

43 Zhang, F. et al. Engineering magnetosomes for ferroptosis/immunomodulation synergism in cancer. ACS Nano 13, 5662-5673 (2019).

44 Porter, A. G. \& Janicke, R. U. Emerging roles of caspase-3 in apoptosis. Cell Death Differ. 6, 99104 (1999).

45 Cadenas, S. Mitochondrial uncoupling, ROS generation and cardioprotection. Biochim. Biophys. Acta, Bioenerg. 1859, 940-950, (2018).

46 Wolkow, C. A. \& Iser, W. B. Uncoupling protein homologs may provide a link between mitochondria, metabolism and lifespan. Ageing Res. Rev. 5, 196-208 (2006).

\section{Figures}


A

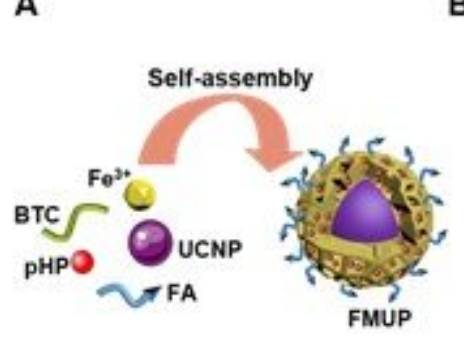

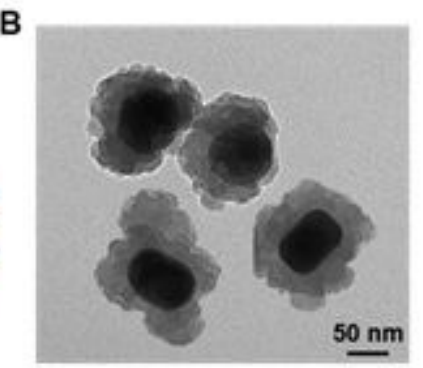
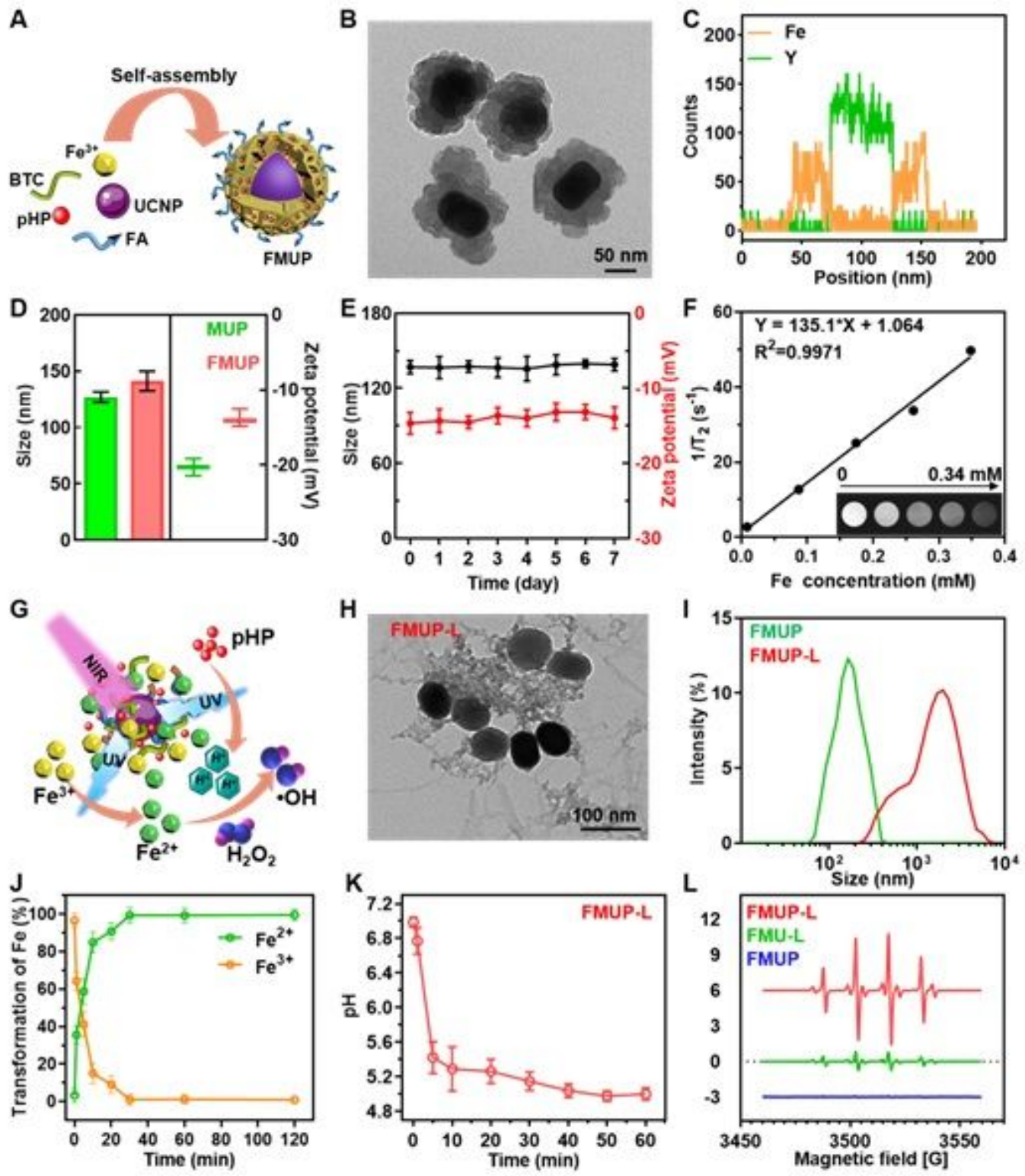

Figure 1

Characterizations of FMUP. (A) Schematic illustration of the construction of FMUP by one-pot selfassembly strategy. (B) TEM image of FMUP. (C) The distribution of Fe element and $\mathrm{Y}$ element in FMUP. (D) Size and zeta potential of FMUP and MUP dispersed in water. (E) The stability of FMUP in cell culture medium. (F) T2 relaxation rate (1/T2, s-1) linearity curve and corresponding T2-weighted MRI images of FMUP with different Fe concentrations. (G) Schematic illustration of nanoagents in response to NIR light irradiation. (H) TEM image of FMUP after NIR irradiation (980 nm, $1.0 \mathrm{~W} / \mathrm{cm} 2,5 \mathrm{~min})$. (I) Size distribution of FMUP dispersed in water before and after NIR irradiation. (J) The transformation of Fe3+ to Fe2+ in FMUP upon continuous NIR irradiation $(980 \mathrm{~nm}, 1.0 \mathrm{~W} / \mathrm{cm} 2)$. (K) Evolution of $\mathrm{pH}$ value of the FMUP suspension sample upon continuous NIR irradiation $(980 \mathrm{~nm}, 1.0 \mathrm{~W} / \mathrm{cm} 2)$. (L) ESR spectra of various groups. The acid microenvironment could catalyse and accelerate the Fenton-reaction for generation more $\cdot O H$. Data in $(D),(E),(J)$, and $(K)$ represent the means $\pm S D(n=3)$. 

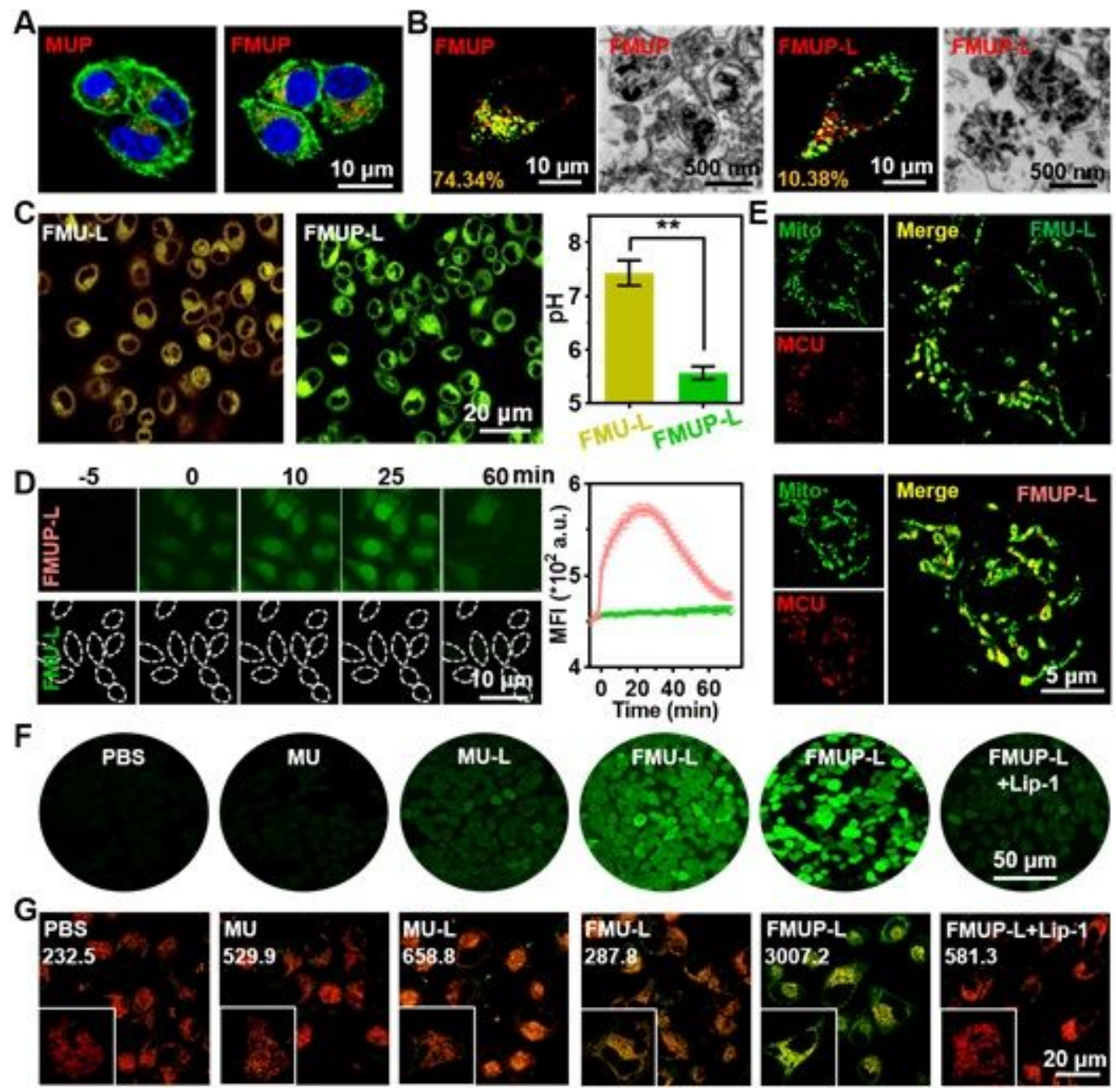

Figure 2

In vitro assessment of the effect of CDT/acidification synergism on mitochondria. (A) CLSM images of MUP and FMUP in HeLa cells. Green: cell membrane; Red: MOFs; Blue: nuclei. (B) CLSM images and TEM images of lysosomal escape of different treatments (FMU-L and FMUP-L). Green: lysosome; Red: MOFs. The number in the lower left corner represents the co-localization rate. (C) CLSM images and further quantitative analysis of $\mathrm{pH}$ value in different groups (FMU-L and FMUP-L). (D) CLSM images of calcium influx in group of FMU-L and FMUP-L. Green: Ca2+. (E) CLSM images of the expression of mitochondrial calcium uniporter (MCU) in the mitochondria. The higher the expression of $\mathrm{MCU}$, the stronger the calcium overload in mitochondria. Green: mitochondrial; Red: MCU. (F) Detection of ROS production in HeLa cytoplasm by DCFH-DA with different treatments. (G) Detection of lipid peroxidation in HeLa cells by Liperfluo with different treatments. Green: lipid peroxidation; Red: mitochondria. Data in (C) and (D) represent the means $\pm S D(n=3)$. Statistical significance between groups in (C) was calculated using twotailed unpaired Student's t-test. ${ }^{*} \mathrm{P}<0.05,{ }^{*} \mathrm{P}<0.01$. 


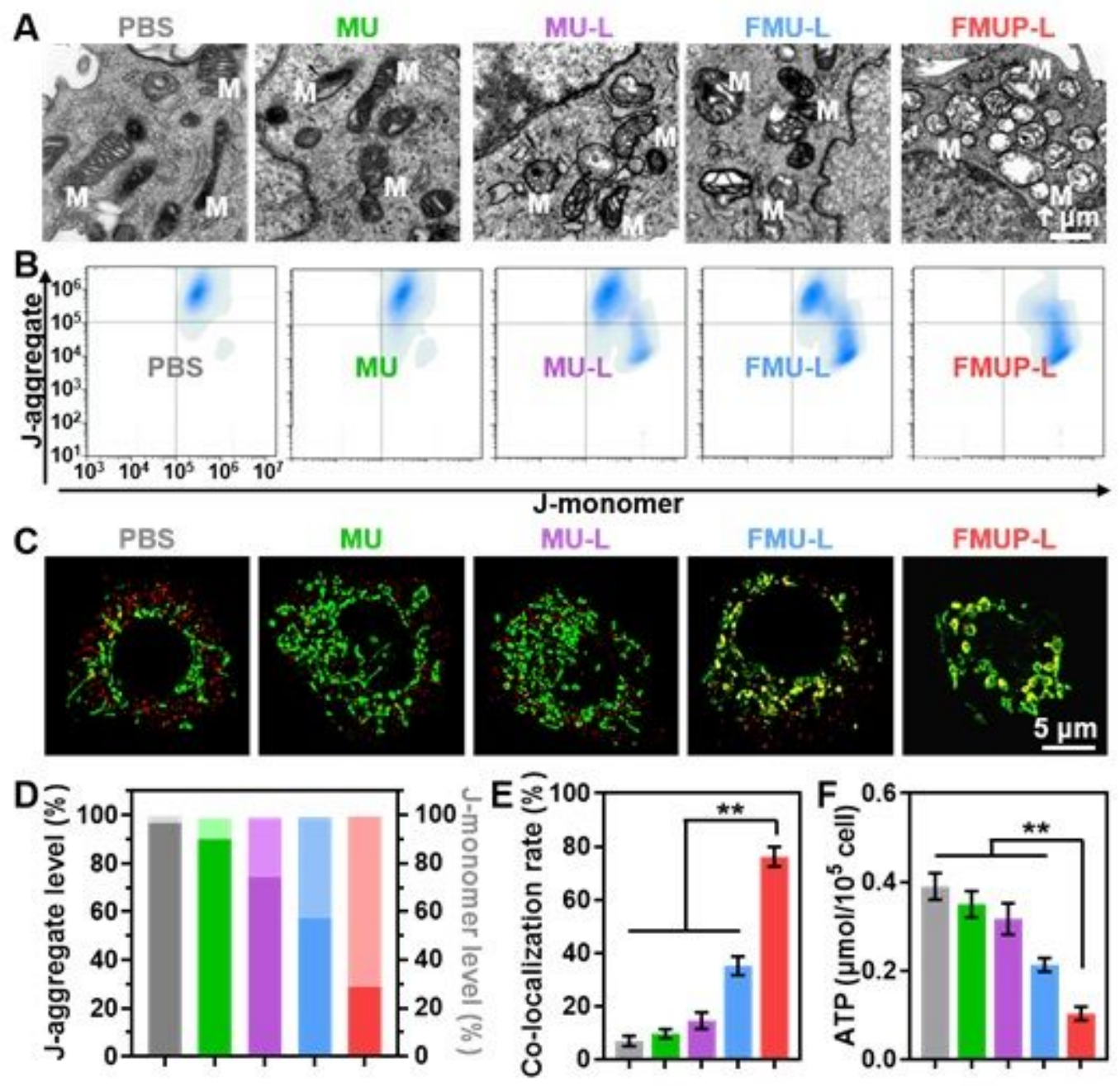

Figure 3

In vitro evaluation of mitochondrial damage by the synergism of CDT and photoacidification. (A) TEM images of mitochondrial integrity in different groups. (B) Mitochondrial membrane potential analysis of HeLa cells treated with different groups by using flow cytometry. (C) CLSM images of cyclophilin D (Cyp D) expression in HeLa cells with different groups. Green: mitochondria; Red: Cyp D. (D) Quantitative analysis of $\mathrm{J}$-aggregate and $\mathrm{J}$-monomer in various treatments. $(\mathrm{E})$ The co-localization rate of nanoagents with mitochondria in different groups. (F) Quantitative analysis of intracellular ATP concentration in various treatments. Data in (D), $(E)$ and $(F)$ represent the means $\pm S D(n=3)$. Statistical significance between multiple groups in $(E)$ and $(F)$ were calculated using one-way ANOVA. ${ }^{*} P<0.05,{ }^{*} P<0.01$. 

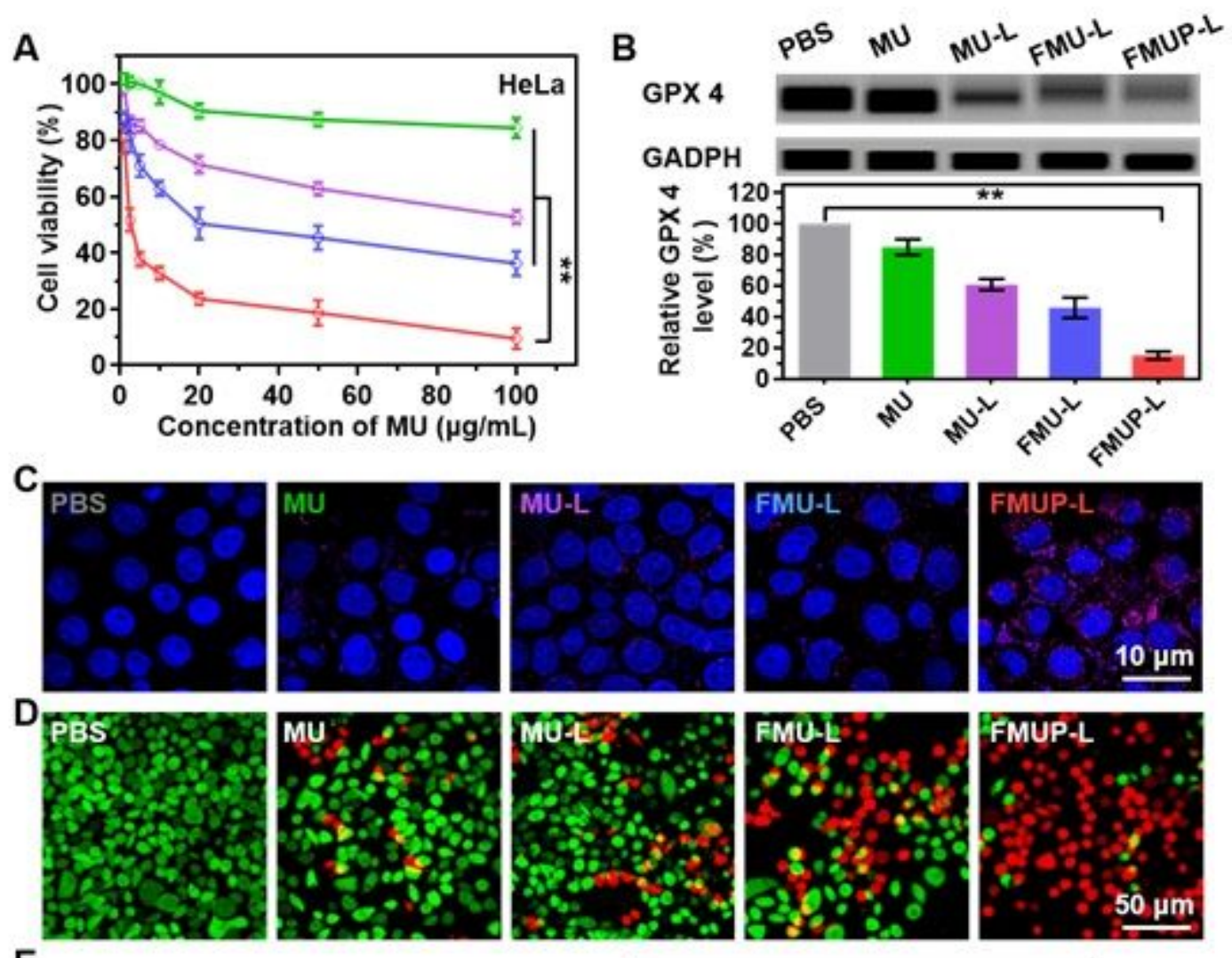

E
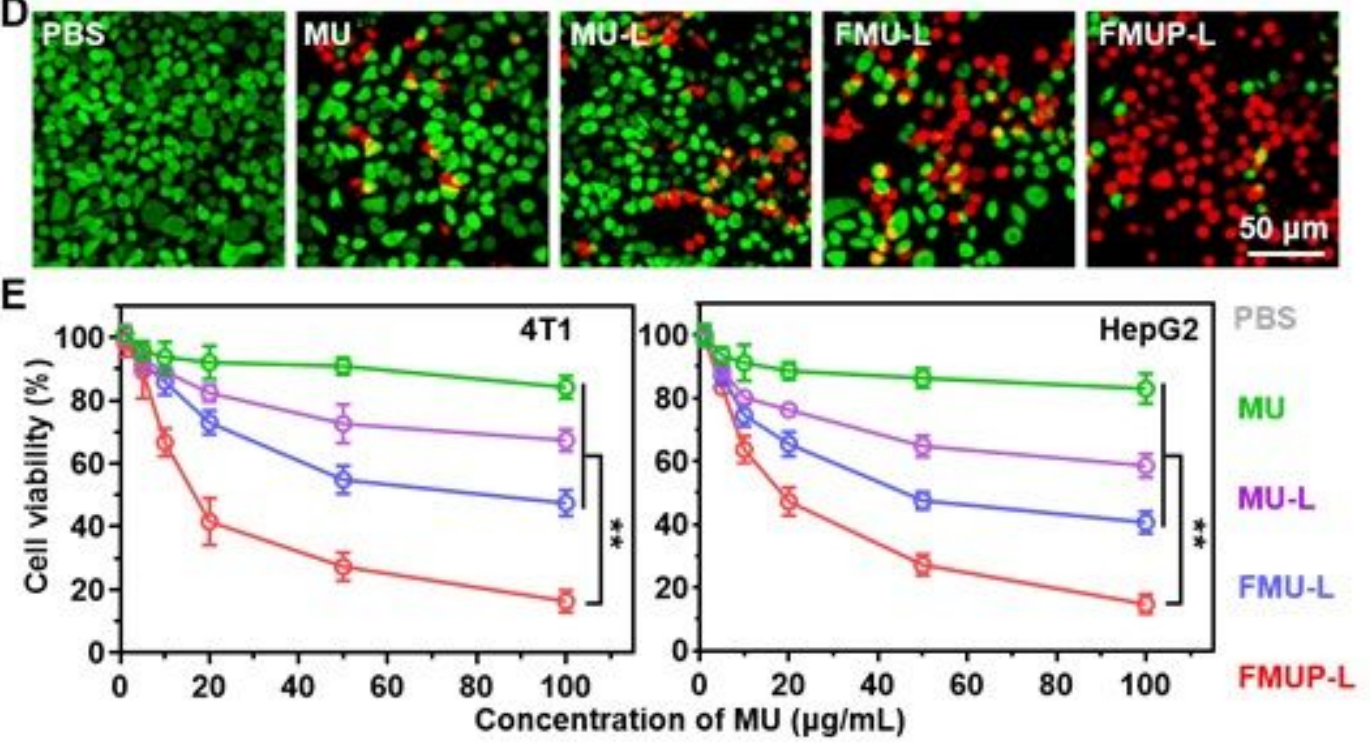

Figure 4

The cytotoxicity of various treatments and the analysis of combined ferroptosis and apoptosis. (A) CCK-8 cytotoxicity analysis of HeLa cells treated with different formulations. (B) Western blotting analysis of glutathione peroxidase (GPX4) by ProteinSimple WesTM Capillary Western Analyzer. (C) The analysis of caspase-3 activity in HeLa cells using Alexa 647-conjugated caspase-3 antibody. Pink: caspase-3; Blue: nuclei. (D) Live/dead cytotoxicity analysis of HeLa cells treated with different formulations. Green: live cells; Red: dead cells. (E) CCK-8 cytotoxicity analysis of 4T1 and HepG2 cells treated with different formulations. Data in $(A),(B)$, and $(E)$ represent mean $\pm S D(n=3)$. Statistical significance between multiple groups in (A), (B) and (E) were calculated using one-way ANOVA. $* P<0.05, * \star P<0.01$. 

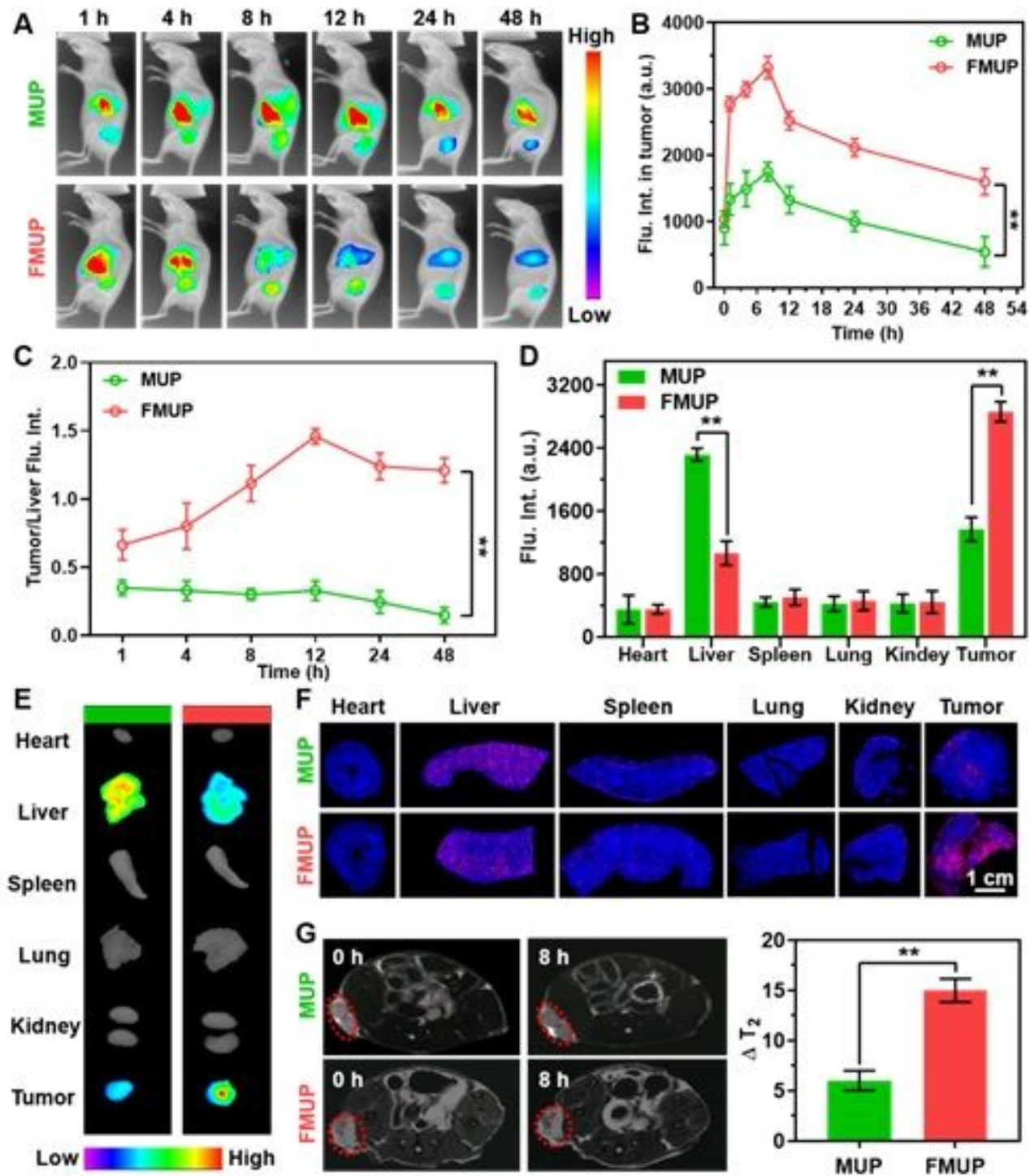

F Heart Liver
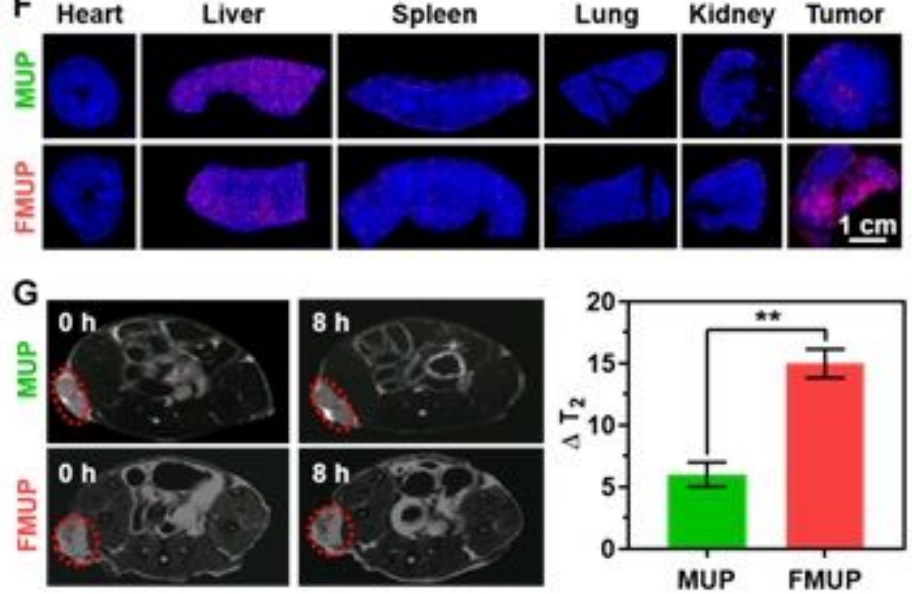

\section{Figure 5}

In vivo biodistribution of MOF-based nanoagents. (A) In vivo fluorescence imaging of biodistribution of MUP and FMUP in HeLa tumor-bearing mice over time. (B) The fluorescence intensity of tumors in the group of MUP and FMUP with elapsing time. (C) The tumor/liver fluorescence intensity ratios in the group of MUP and FMUP with elapsing time. (D) Ex vivo images of tumors and other main organs at $48 \mathrm{~h}$ postinjection and corresponding fluorescence intensity analysis in the group of MUP and FMUP. (F) Frozen section staining images of main organs and tumors in MUP group and FMUP group. Pink: Cy 5lablled MOFs. Blue: nuclei. (G) T2-MRI images of mice before and after injection and corresponding analysis of T2 value in the group of MUP and FMUP. Red dotted circles indicate the tumors. Data in (B), $(C),(D)$ and $(G)$ represent mean $\pm S D(n=3)$. Statistical significance between groups in (B), (C), (D), and (G) were calculated using two-tailed unpaired Student's t-test. ${ }^{\star} P<0.05,{ }^{\star} * \mathrm{P}<0.01$. 
A
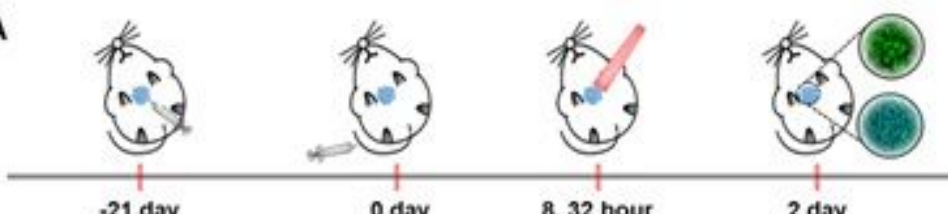

8,32 hour

-21 day
HeLa tumor

inoculation
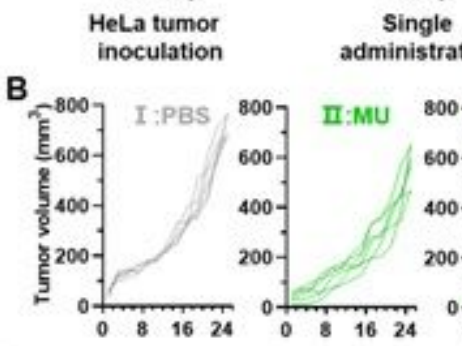

Twice laser

$\mathrm{pH}, \mathrm{Ca}^{2+}, \mathrm{ROS}$

and IHC analysis

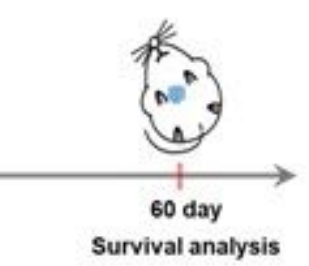

C
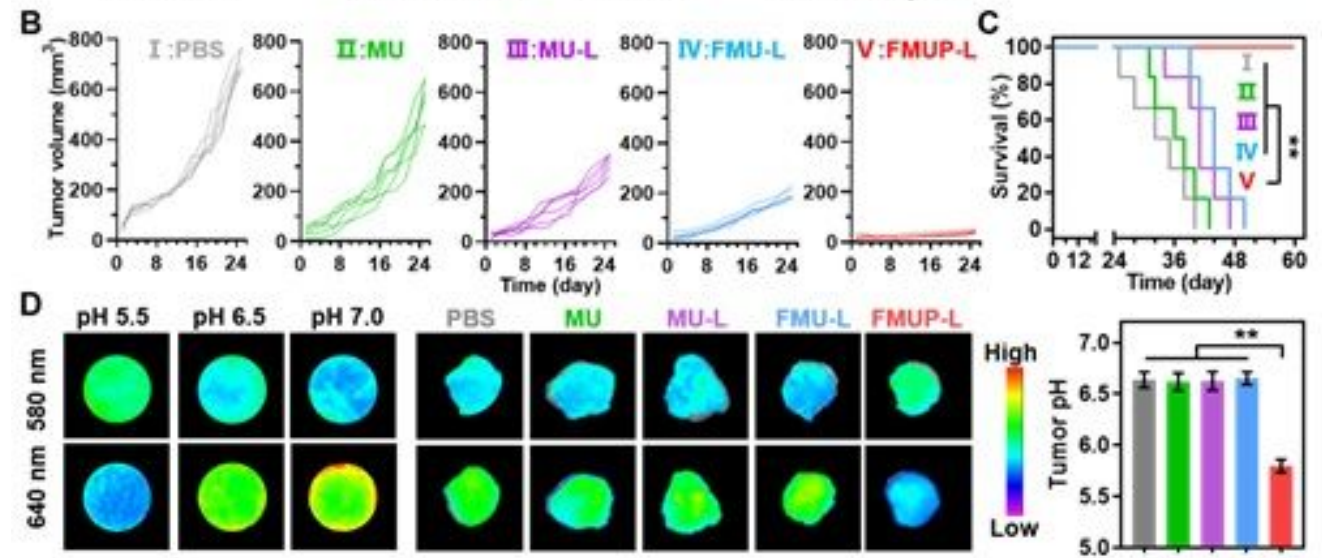

$\mathrm{E}$
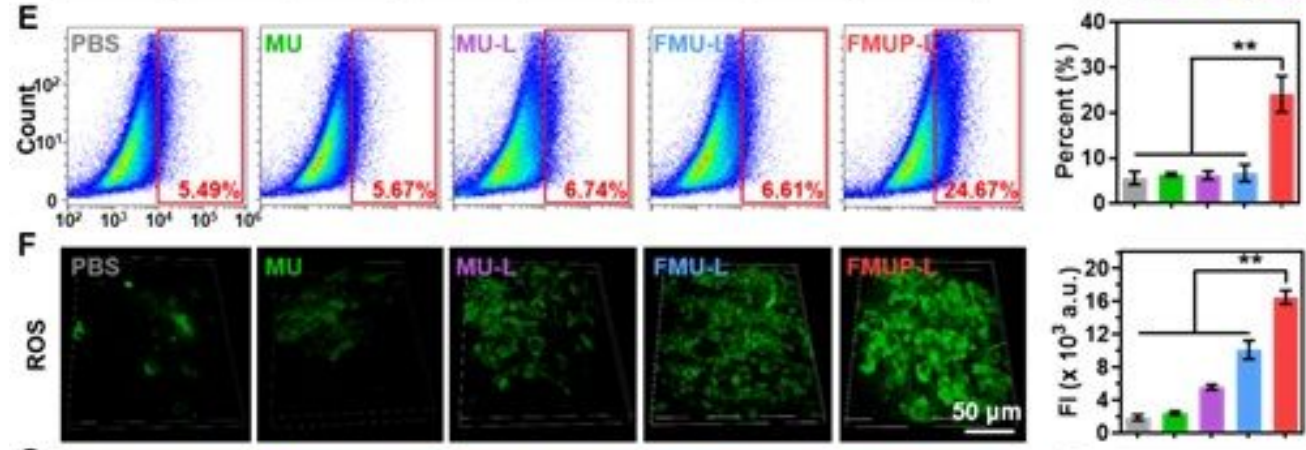

G
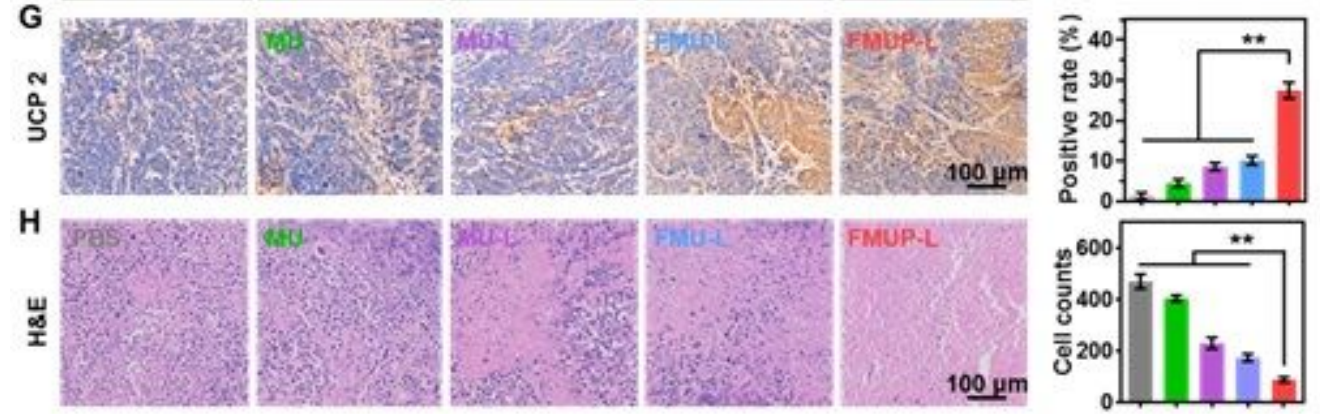

\section{Figure 6}

In vivo antitumor effects in HeLa-cell-derived tumor xenograft model. (A) Schematic illustration of anticancer therapy for HeLa primary tumors. (B) Tumor growth curves of different treatments over 26 days $(n=6)$. (C) Survival curves of tumor bearing mice treated with different groups. (D) Fluorescence imaging of tumor $\mathrm{pH}$ value in different groups by using SNARF®-1 and corresponding quantification analysis. (E) The analysis of calcium influx in tumors with different treatments by using flow cytometry and corresponding analysis. (F) Two-photon fluorescence imaging and corresponding fluorescence intensity analysis of ROS in tumors in different treatments by using DCFH-DA. (G) Immunohistochemical staining images and further quantitative analysis of UCP 2 in tumor tissue for revealing the mitochondrial damage. $(\mathrm{H})$ Hematoxylin and eosin (H\&E) staining images of tumors in different groups and corresponding quantitative analysis for tumor cells. Data in (B) and (C) represent mean $\pm S D(n=6)$. Data 
in $(D-H)$ represent mean $\pm S D(n=3)$. Statistical significance between multiple groups in $(D),(F),(G)$ and $(\mathrm{H})$ were calculated using one-way ANOVA and survival analysis in (C) was calculated by Log-rank test. * $\mathrm{P}<0.05$, ** $\mathrm{P}<0.01$.

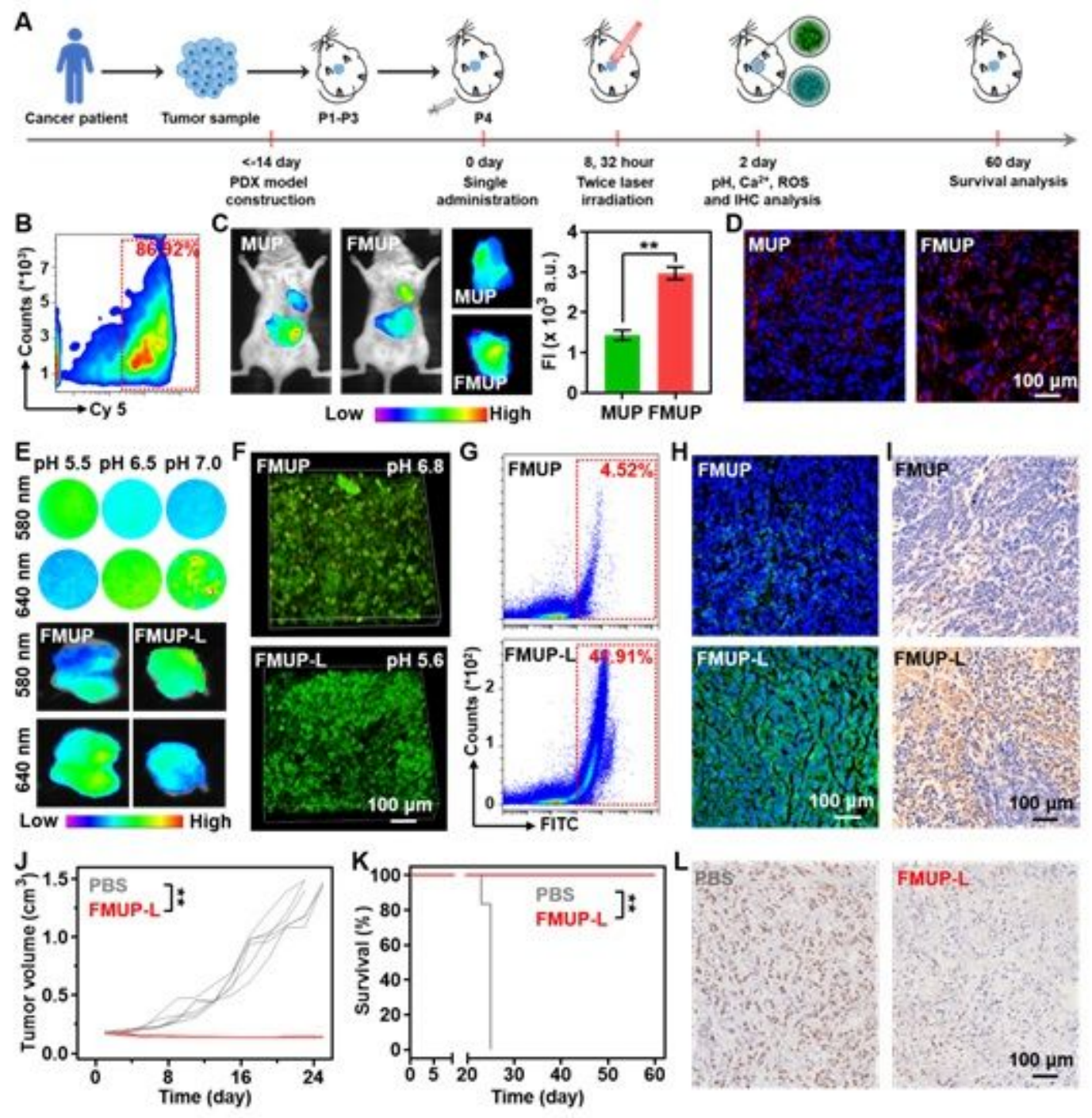

Figure 7

In vivo antitumor effects in patient-derived tumor xenograft (PDX) model. (A) Schematic illustration of procedure for evaluating the antitumor activity in liver-cancer-PDX model. (B) The analysis of FA receptor in human hepatoma cells via flow cytometry. (C) In vivo fluorescence imaging of biodistribution of MUP and FMUP in model mice $8 \mathrm{~h}$ after injection and the corresponding quantitative analysis for various tumors. (D) Frozen section staining images of tumors in the group of MUP and FMUP. Red: Cy 5-lablled MOFs; Blue: nuclei. (E) Fluorescence imaging of tumor $\mathrm{pH}$ in the group of MUP and FMUP using SNARF®-1. (F) Two-photon fluorescence imaging of tumor $\mathrm{pH}$ in the group of FMUP and FMUP-L using SNARFß-1. (G) The analysis of ROS in tumors in the group of FMUP and FMUP-L via flow cytometry using DCFH-DA as the probe. $(\mathrm{H})$ Frozen section staining imaging of tumors for evaluating the calcium influx after different treatments using Fluo-3 as the probe. (I) Immunohistochemical staining images and further quantitative analysis of UCP 2 in tumor tissue for revealing the mitochondrial damage. (J) Tumor 
growth curves of different treatments over 26 days $(n=6)$. (K) Survival curves of tumor-bearing mice in the group of PBS and FMUP-L. (L) Representative cell proliferation analysis of tumor tissue by Ki 67 method. Data in (C) represent mean $\pm S D(n=3)$. Data in $(J)$ and $(K)$ represent mean $\pm S D(n=6)$. Statistical significance between groups in $(\mathrm{C})$ and $(\mathrm{K})$ were calculated using two-tailed unpaired Student's t-test. ${ }^{\star} \mathrm{P}<0.05,{ }^{*} \mathrm{P}<0.01$.

\section{Supplementary Files}

This is a list of supplementary files associated with this preprint. Click to download.

- SupplementaryInfoMOFbasedanticancernanoagent.docx

- Scheme1.pdf 